\title{
The Higgs sector of the phenomenological MSSM in the light of the Higgs boson discovery
}

\author{
A. Arbey, ${ }^{a, b, c}$ M. Battaglia, ${ }^{c, d, e}$ A. Djouadi ${ }^{c, f}$ and F. Mahmoudi ${ }^{c, g}$ \\ ${ }^{a}$ Université de Lyon, France, Université Lyon 1, \\ CNRS/IN2P3, UMR5822 IPNL, F-69622 Villeurbanne Cedex, France \\ ${ }^{b}$ Centre de Recherche Astrophysique de Lyon, \\ Observatoire de Lyon, Ecole Normale Supérieure de Lyon, \\ Saint-Genis Laval Cedex, CNRS, UMR 5574, F-69561, France \\ ${ }^{c}$ CERN, \\ CH-1211 Geneva 23, Switzerland \\ ${ }^{d}$ Santa Cruz Institute of Particle Physics, University of California, \\ Santa Cruz, CA 95064, U.S.A. \\ ${ }^{e}$ Lawrence Berkeley National Laboratory, \\ Berkeley, CA 94720, U.S.A. \\ ${ }^{f}$ Laboratoire de Physique Théorique, Université Paris XI and CNRS, \\ F-91405 Orsay, France \\ ${ }^{g}$ Clermont Université, Université Blaise Pascal, \\ CNRS/IN2P3, LPC, BP 10448, 63000 Clermont-Ferrand, France
}

E-mail: Alexandre.Arbey@ens-lyon.fr, Marco.Battaglia@cern.ch, Abdelhak.Djouadi@cern.ch, Mahmoudi@in2p3.fr

ABSTRACT: The long awaited discovery of a new light scalar boson at the LHC opens up a new era of studies of the Higgs sector in the Standard Model and in its extensions. In this paper we discuss the consequences of the observation of a light Higgs boson with the mass and rates reported by the ATLAS and CMS collaborations on the parameter space of the phenomenological MSSM, accounting also for the LHC searches for heavier Higgs bosons and supersymmetric particle partners, as well as constraints from $B$-physics and dark matter. We explore the various regimes of the MSSM Higgs sector, depending on the parameters $M_{A}$ and $\tan \beta$, and show that only two of them are still allowed by all present experimental constraints: the decoupling regime, in which there is only one light and standard-like Higgs boson while the heavier Higgs states decouple from gauge bosons, and the supersymmetric regime, in which there are light supersymmetric particle partners which might affect the decay properties of the light Higgs boson, in particular its di-photon and invisible decays.

KeYwords: Supersymmetry Phenomenology

ARXIV EPRINT: 1207.1348 


\section{Contents}

1 Introduction 1

2 The theoretical set-up $\quad 2$

2.1 The pMSSM Higgs sector 2

2.2 The various regimes of the pMSSM 4

$\begin{array}{lll}2.3 & \text { Higgs decays and production in the pMSSM } & 6\end{array}$

3 Analysis and results $\quad 9$

3.1 pMSSM scans and software tools $\quad 9$

$\begin{array}{lll}3.2 & \text { Constraints } & 10\end{array}$

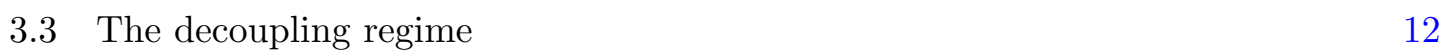

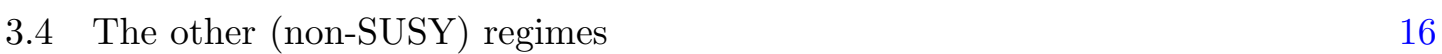

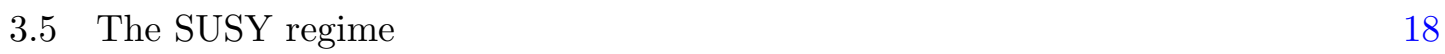

$\begin{array}{lll}\text { 3.5.1 Invisible Higgs decays } & 18\end{array}$

$\begin{array}{ll}\text { 3.5.2 Sparticle effects on the } h b \bar{b} \text { coupling } & 19\end{array}$

3.5.3 Sparticle contributions to the hgg and $\mathrm{h} \gamma \gamma$ vertices 21

3.5.4 Impact of the LHC data 22

4 Conclusions $\quad 24$

\section{Introduction}

Results of the search for the Higgs bosons at the LHC with the $10 \mathrm{fb}^{-1}$ data collected in 2011 at $7 \mathrm{TeV}$ and 2012 at $8 \mathrm{TeV}$ have just been presented by the ATLAS [1,2] and CMS $[3,4]$ collaborations and there is now a $5 \sigma$ evidence by each of the experiments for a new particle with a mass of $\approx 126 \mathrm{GeV}$. Complementary evidence is also provided by the updated combination of the Higgs searches performed by the CDF and D0 collaborations at the Tevatron [5], which has also been just released. As we are entering an era of Higgs studies, these results have deep implications for the Minimal Supersymmetric Standard Model (MSSM). The implications of a Higgs boson with a mass value around $126 \mathrm{GeV}$ in the context of Supersymmetry have been already widely discussed [6-17] since the first evidence of a signal at the LHC was presented at the end of 2011. In particular, we have discussed the consequences of the value of $M_{h}$ for the unconstrained phenomenological MSSM (pMSSM) with 22 free parameters [18], for constrained MSSM scenarios such as the minimal gravity, gauge and anomaly mediated SUSY-breaking models, and in scenarios in which the supersymmetric spectrum is extremely heavy [6]. We have shown that only when the SUSY-breaking scale is very large or the mixing in the stop sector is significant, the observed $M_{h}$ value can be accommodated in these models. This disfavours many 
constrained scenarios, such as the minimal anomaly and gauge mediated SUSY-breaking models, and some, even more constrained, versions of the minimal super-gravity model.

In this paper, we extend the previous study in new directions afforded by the new data from the LHC experiments. First, we refine our analysis of the implications of the value $M_{h} \approx 126 \mathrm{GeV}$ for the decoupling regime by considering different types of stop mixing scenarios, which significantly affect the maximal mass value, and we explore the implications of a broader range of the top quark mass value, $m_{t}=(173 \pm 3) \mathrm{GeV}$, on $M_{h}$. Then, we analyse in details the implications of the ATLAS and CMS searches for the heavier MSSM Higgs bosons, the CP-even $H$, a pseudoscalar $A$ and two charged $H^{ \pm}$states. In particular, we discuss the $A / H / h \rightarrow \tau^{+} \tau^{-}$for the neutral and the $t \rightarrow b H^{+} \rightarrow b \tau \nu$ searches for the charged states to further constrain the $\left[M_{A}, \tan \beta\right]$ parameter space, where $\tan \beta$ is the ratio of the vacuum expectation values of the two Higgs doublet fields. We also discuss the effect of the recent LHCb results for the decay $B_{s} \rightarrow \mu^{+} \mu^{-}$on the Higgs sector, as well as the super-particle LHC searches and the dark matter constraints. Most importantly, we study other regimes than the decoupling regime of the pMSSM: the antidecoupling regime for low $M_{A}$ in which the roles of the $h$ and $H$ bosons are reversed, the intense coupling regime, in which the three neutral particles $h, A, H$ are rather close in mass, the intermediate regime at relatively low $\tan \beta$, in which the couplings of $H, A$ to gauge + Higgs bosons are not too suppressed, and the vanishing coupling regime, in which the coupling of the $h$ state to bottom quarks or gauge bosons is suppressed. Using the latest ATLAS, CMS and Tevatron data, we show that all these scenarios are now almost ruled out. Finally, we start studying the implications of the rates reported by the LHC experiments in the $\gamma \gamma$ and $Z Z$ final states used to obtain the Higgs boson signal and we comment on the $b \bar{b}$ final state, to which the Tevatron is most sensitive. We perform a full scan of the pMSSM parameter space in order to delineate the regions which fit best the experimental data, including a possible enhancement of the $h \rightarrow \gamma \gamma$ rate.

The paper is organised as follows. First, we briefly describe the pMSSM and its Higgs sector with its various regimes and summarise the Higgs decays and the production cross sections at the LHC. In section 3, we present the analysis of these different Higgs regimes and the implications on the pMSSM parameters in the light of the LHC Higgs discovery and constraints. Section 4 has a short conclusion.

\section{The theoretical set-up}

\subsection{The pMSSM Higgs sector}

In the MSSM the Higgs sector is extended to contain five Higgs particles. ${ }^{1}$ The lightest $h$ boson has in general the properties of the Standard Model (SM) Higgs boson ${ }^{2}$ and is expected to have a mass $M_{h} \lesssim 115-135 \mathrm{GeV}$ depending on the MSSM parameters, in particular, the ratio $\tan \beta$. By virtue of supersymmetry, only two parameters are needed to describe the Higgs sector at tree-level. These can be conveniently chosen to be the

\footnotetext{
${ }^{1}$ For a review of the MSSM Higgs sector [19]. For reviews on the radiative corrections in the MSSM Higgs sector and a complete set of references, see [20-22].

${ }^{2}$ For a review of the SM Higgs boson, see [23].
} 
pseudoscalar boson mass $M_{A}$ and the ratio of vacuum expectation values of the two Higgs fields that break the symmetry, $\tan \beta=v_{2} / v_{1}$. However, accounting for the radiative corrections to the Higgs sector, known to play an extremely important role [20-22], all soft SUSY-breaking parameters which are of $\mathcal{O}(100)$ in addition to those of the SM, become relevant. This makes any phenomenological analysis in the most general MSSM a very complicated task. A phenomenologically more viable MSSM framework, the pMSSM, is defined by adopting the following assumptions: $i$ ) all soft SUSY-breaking parameters are real and there is no new source of CP-violation; $i i)$ the matrices for the sfermion masses and for the trilinear couplings are all diagonal, implying no flavor change at tree-level; and ii) the soft SUSY-breaking masses and trilinear couplings of the first and second sfermion generations are the same at the electro-weak symmetry breaking scale. Making these three assumptions will lead to only 22 input parameters in the pMSSM:

$-\tan \beta$ : the ratio of the vevs is expected to lie in the range $1 \lesssim \tan \beta \lesssim m_{t} / m_{b}$;

- $M_{A}$ : the pseudoscalar Higgs mass that ranges from $M_{Z}$ to the SUSY-breaking scale;

— $\mu$ : the Higgs-higgsino (supersymmetric) mass parameter (with both signs);

- $M_{1}, M_{2}, M_{3}$ : the bino, wino and gluino mass parameters;

- $m_{\tilde{q}}, m_{\tilde{u}_{R}}, m_{\tilde{d}_{R}}, m_{\tilde{l}}, m_{\tilde{e}_{R}}:$ the first/second generation sfermion mass parameters;

- $A_{u}, A_{d}, A_{e}$ : the first/second generation trilinear couplings;

$-m_{\tilde{Q}}, m_{\tilde{t}_{R}}, m_{\tilde{b}_{R}}, m_{\tilde{L}}, m_{\tilde{\tau}_{R}}:$ the third generation sfermion mass parameters;

- $A_{t}, A_{b}, A_{\tau}$ : the third generation trilinear couplings.

Such a model has more predictability and it offers an adequate framework for phenomenological studies. In general, only a small subset of the parameters appears when looking at a given sector of the pMSSM, such as the Higgs sector in this case. Some of these parameters will enter the radiative corrections to the Higgs boson masses and couplings. At the one-loop level, the $h$ boson mass receives corrections that grow as the fourth power of the top quark mass $m_{t}$ (we use the running $\overline{\mathrm{MS}}$ mass to re-sum some higher order corrections) and logarithmically with the SUSY-breaking scale or common squark mass $M_{S}$; the trilinear coupling in the stop sector $A_{t}$ plays also an important role. The leading part of these corrections reads [24-26]

$$
\epsilon=\frac{3 \bar{m}_{t}^{4}}{2 \pi^{2} v^{2} \sin ^{2} \beta}\left[\log \frac{M_{S}^{2}}{\bar{m}_{t}^{2}}+\frac{X_{t}^{2}}{2 M_{S}^{2}}\left(1-\frac{X_{t}^{2}}{6 M_{S}^{2}}\right)\right] .
$$

We have defined the SUSY-breaking scale $M_{S}$ to be the geometric average of the two stop masses (that we take $\lesssim 3 \mathrm{TeV}$ not to introduce excessive fine-tuning)

$$
M_{S}=\sqrt{m_{\tilde{t}_{1}} m_{\tilde{t}_{2}}}
$$


and introduced the mixing parameter $X_{t}$ in the stop sector (that we assume $\lesssim 3 M_{S}$ ),

$$
X_{t}=A_{t}-\mu \cot \beta \text {. }
$$

The radiative corrections have a much larger impact and maximise the $h$ boson mass in the so-called "maximal mixing" scenario, where the trilinear stop coupling in the $\overline{\mathrm{DR}}$ scheme is

$$
\text { maximal mixing scenario : } X_{t}=\sqrt{6} M_{S} \text {. }
$$

In turn, the radiative corrections are much smaller for small values of $X_{t}$, i.e. in the

$$
\text { no mixing scenario: } \quad X_{t}=0 \text {. }
$$

An intermediate scenario is when $X_{t}$ is of the same order as $M_{S}$ which is sometimes called the

$$
\text { typical mixing scenario : } X_{t}=M_{S} \text {. }
$$

These mixing scenarios have been very often used as benchmarks for the analysis of MSSM Higgs phenomenology [27]. The maximal mixing scenario has been particularly privileged since it gives a reasonable estimate of the upper bound on the $h$ boson mass, $M_{h}^{\max }$. We will discuss these scenarios but, compared to the work of ref. [27], we choose here to vary the scale $M_{S}$. Together with the requirements on $X_{t}$ in eqs. (4-6), we adopt the following values for the parameters entering the pMSSM Higgs sector,

$$
A_{t}=A_{b}, M_{2} \simeq 2 M_{1}=|\mu|=\frac{1}{5} M_{S}, M_{3}=0.8 M_{S},
$$

and vary the basic inputs $\tan \beta$ and $M_{A}$. For the values $\tan \beta=60$ and $M_{A}=M_{S}=3 \mathrm{TeV}$ and a top quark pole of mass of $m_{t}=173 \mathrm{GeV}$, we would obtain a maximal Higgs mass value $M_{h}^{\max } \approx 135 \mathrm{GeV}$ for maximal mixing, once the full set of known radiative corrections up to two loops is implemented [28]. In the no-mixing and typical mixing scenarios, we obtain much smaller values, $M_{h}^{\max } \approx 120 \mathrm{GeV}$ and $M_{h}^{\max } \approx 125 \mathrm{GeV}$, respectively. Scanning over the soft SUSY-breaking parameters, one may increase these $M_{h}^{\max }$ values by up to a few $\mathrm{GeV}$.

It is important to note that the dominant two-loop corrections have been calculated in the $\overline{\mathrm{DR}}$ scheme [29-31] and implemented in the codes Suspect [32] and SOFTSUSY [33] that we will use here for the MSSM spectrum, but also in the on-shell scheme [34, 35], implemented in FeynHiggs [36]. In general, the results for $M_{h}$ in the two schemes differ by at most $2 \mathrm{GeV}$, which we take as a measure of the missing higher order effects. Quite recently, the dominant three-loop contribution to $M_{h}$ has been calculated and found to be below $1 \mathrm{GeV}$ [37]. Thus, the mass of the lightest $h$ boson can be predicted with an accuracy of $\Delta M_{h} \sim 3 \mathrm{GeV}$ and this is the theoretical uncertainty on $M_{h}$ that we assume.

\subsection{The various regimes of the pMSSM}

The spectrum in the various regimes of the pMSSM Higgs sector [19], depends on the values of $M_{A}$ and also on $\tan \beta$, and we shall confront it to the latest LHC and Tevatron data in the following. 
We start from the decoupling regime $[38,39]$ that has been already mentioned and which in principle occurs for large values of $M_{A}$ but is reached in practice at $M_{A} \gtrsim 300 \mathrm{GeV}$ for low $\tan \beta$ values and already at $M_{A} \gtrsim M_{h}^{\max }$ for $\tan \beta \gtrsim 10$. In this case, the CP-even $h$ boson reaches its maximal mass value $M_{h}^{\max }$ and its couplings to fermions and gauge bosons (as well as its self-coupling) become SM-like. The heavier $H$ boson has approximately the same mass as the $A$ boson and its interactions are similar, i.e. its couplings to gauge bosons almost vanish and the couplings to bottom (top) quarks and $\tau$ leptons fermions are (inversely) proportional to $\tan \beta$. Hence, one will have a SM-like Higgs boson $h \equiv H_{\mathrm{SM}}$ and two pseudo-scalar (like) Higgs particles, $\Phi=H, A$. The $H^{ \pm}$boson is also degenerate in mass with the $A$ boson and the intensity of its couplings to fermions is similar. Hence, in the decoupling limit, the heavier $H / A / H^{ \pm}$bosons almost decouple and the MSSM Higgs sector reduces effectively to the SM Higgs sector, but with a light $h$ boson.

The anti-decoupling regime [40] occurs for a light pseudo-scalar Higgs boson, $M_{A} \lesssim M_{h}^{\max }$, and is exactly opposite to the decoupling regime. The roles of the $h$ and $H$ bosons are reversed and at large $\tan \beta$ values, the $h$ boson is degenerate in mass with the pseudo-scalar $A, M_{h} \simeq M_{A}$, while the $H$ boson has a mass close to its minimum, which is in fact $M_{h}^{\max }$. Because of the upper bound on $M_{h}$, all Higgs particles are light. Here, it is the $h$ boson which has couplings close to those of $A, \Phi=h, A$, while the $H$ boson couplings are SM-like, $H \equiv H_{\mathrm{SM}}$.

The intense-coupling regime [41-44] occurs when the mass of the pseudo-scalar $A$ boson is close to $M_{h}^{\max }$. In this case, the three neutral Higgs bosons $h, H$ and $A$ (as well as the charged Higgs particles) have comparable masses, $M_{h} \sim M_{H} \sim M_{A} \sim M_{h}^{\max }$. This mass degeneracy is more effective when $\tan \beta$ is large. Here, both the $h$ and $H$ bosons have still enhanced couplings to $b$-quarks and $\tau$ leptons and suppressed couplings to gauge bosons and top quarks, as the pseudo-scalar $A$. Hence, one approximately has three pseudo-scalar like Higgs particles, $\Phi \equiv h, H, A$ with mass differences of the order of $10-20 \mathrm{GeV}$.

The intermediate-coupling regime occurs for low values of $\tan \beta, \tan \beta \lesssim 5$-10, and a not too heavy pseudo-scalar Higgs boson, $M_{A} \lesssim 300-500 \mathrm{GeV}[19]$. Hence, we are not yet in the decoupling regime and both CP-even Higgs bosons have non-zero couplings to gauge bosons and their couplings to down-type (up-type) fermions (as is the case for the pseudoscalar $A$ boson) are not strongly enhanced (suppressed) since $\tan \beta$ is not too large. This scenario is already challenged by LEP2 data which call for moderately large values of $\tan \beta$.

The vanishing-coupling regime occurs for relatively large values of $\tan \beta$ and intermediate to large $M_{A}$ values, as well as for specific values of the other MSSM parameters. The latter parameters, when entering the radiative corrections, could lead to a strong suppression of the couplings of one of the CP-even Higgs bosons to fermions or gauge bosons, as a result of the cancellation between tree-level terms and radiative corrections [45-50]. An example of this situation is the small $\alpha_{\text {eff }}$ scenario, used as a benchmark [27] in which the Higgs coupling to $b \bar{b}$ is strongly suppressed.

The parameter space, within the plane $\left[M_{A}, \tan \beta\right]$, in which the above regimes of the pMSSM Higgs sector occur is shown in figure 1. We have chosen the usual maximal mixing scenario with $M_{S}=2 \mathrm{TeV}$ and the other SUSY parameters as in eq. (2.7). We take 


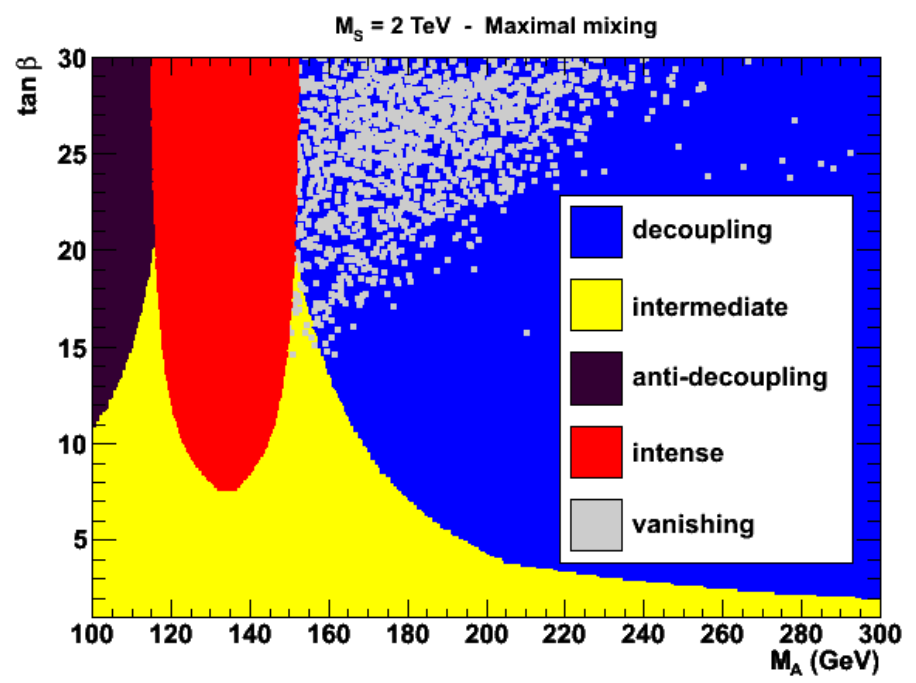

Figure 1. The parameter space for the various regimes of the MSSM Higgs sector as defined in the text and in eq. (8) in the $\left[M_{A}, \tan \beta\right]$ plane; the maximal mixing scenario with $M_{S}=2 \mathrm{TeV}$ is adopted.

exception for the vanishing coupling scenario, where we perform a scan over the pMSSM parameters, and only $\approx 5 \times 10^{-4}$ of the scanned points fulfil its requirements. The following conditions have been imposed:

$$
\begin{gathered}
\text { decoupling regime }: \cos ^{2}(\beta-\alpha) \leq 0.05 \\
\text { anti - decoupling regime }: \cos ^{2}(\beta-\alpha) \geq 0.95 \\
\text { intermediate - coupling regime }: 0.05 \leq \cos ^{2}(\beta-\alpha) \leq 0.7, \quad \tan \beta \leq 10 \\
\text { intense - coupling regime }: M_{A} \lesssim 140 \mathrm{GeV}, g_{h b b}^{2} \text { and } g_{H b b}^{2} \geq 50 \\
\text { vanishing - coupling regime }: M_{A} \gtrsim 200 \mathrm{GeV}, g_{h b b}^{2} \text { or } g_{h V V}^{2} \leq 0.05 .
\end{gathered}
$$

Finally, we consider the SUSY regime, in which some SUSY particles, such as the charginos, neutralinos as well as the third generation sleptons and squarks, could be light enough to significantly affect the phenomenology of the MSSM Higgs bosons. For instance, light sparticles could substantially contribute to the loop induced production and decays modes of the lighter $h$ boson $[51,52]$ and could even appear (in the case of the lightest neutralino) in its decay product as discussed below, in 3.5.1.

\subsection{Higgs decays and production in the pMSSM}

For the relatively large values of $\tan \beta$ presently probed at the LHC, $\tan \beta \gtrsim 7$ as discussed below, the couplings of the non-SM like Higgs bosons to $b$ quarks and $\tau$ leptons are strongly enhanced and those to top quarks and gauge bosons suppressed. The pattern becomes as simple as the following, with more details given in ref. [19]:

— The $\Phi=A$ or $H / h$ bosons in the decoupling/anti-decoupling limit decay almost exclusively into $b \bar{b}$ and $\tau^{+} \tau^{-}$pairs, with branching ratios of, respectively, $\approx 90 \%$ and 
$\approx 10 \%$, and all other channels are suppressed to a level where their branching ratios are negligible.

- The $H^{ \pm}$particles decay into fermion pairs: mainly $H^{+} \rightarrow t \bar{b}$ and $H^{+} \rightarrow \tau \nu_{\tau}$ final states for $H^{ \pm}$masses, respectively, above and below the $t b$ threshold.

- The CP-even $h$ or $H$ boson, depending on whether we are in the decoupling or antidecoupling regime, will have the same decays as the SM Higgs boson. For $M_{h / H} \approx$ $126 \mathrm{GeV}$, the main decay mode will be the $b \bar{b}$ channel with a $\sim 60 \%$ probability, followed by the decays into $c \bar{c}, \tau^{+} \tau^{-}$and the loop induced decay into gluons with $\sim$ $5 \%$ branching ratios. The $W W^{*}$ decay reaches the level of $20 \%$, while the rate for $Z Z^{*}$ is a few times $10^{-2}$. The important loop induced $\gamma \gamma$ decay mode, which leads to most significant signals at the $\mathrm{LHC}$, have rates of $\mathcal{O}\left(10^{-3}\right)$.

In the intense-coupling regime, the couplings of both $h$ and $H$ to gauge bosons and up-type fermions are suppressed and those to down-type fermions are enhanced. The branching ratios of the $h$ and $H$ bosons to $b \bar{b}$ and $\tau^{+} \tau^{-}$final states are thus the dominant ones, with values as in the case of the pseudoscalar $A$ boson. In the intermediate-coupling regime, interesting decays of $H, A$ and $H^{ \pm}$into gauge and/or Higgs bosons occur, as well as $A / H \rightarrow t \bar{t}$ decays, but they are suppressed in general. Finally, for the rare vanishingcoupling regime when the Higgs couplings to $b$-quarks and eventually $\tau$-leptons accidentally vanish, the outcome is spectacular for the $h$ boson: the $W W^{*}$ mode becomes dominant and followed by $h \rightarrow g g$, while the interesting $h \rightarrow \gamma \gamma$ and $h \rightarrow Z Z^{*}$ decay modes are enhanced.

In the case of the SM-like Higgs particle (that we assume now to be the $h$ boson), there are two interesting scenarios which might make its decays rather different. First we have the scenario with the Higgs bosons decaying into supersymmetric particles. Because most sparticles must be heavier than about $100 \mathrm{GeV}$, there is no SUSY decays of the $h$ boson except for the invisible channel into a pair of the lightest neutralinos, $h \rightarrow \chi_{1}^{0} \chi_{1}^{0}$. This is particularly true when the gaugino mass universality relation $M_{2} \sim 2 M_{1}$ is relaxed, leading to light $\chi_{1}^{0}$ states while the LEP2 bound, $m_{\chi_{1}^{ \pm}} \gtrsim 100 \mathrm{GeV}$, still holds. In the decoupling limit, the branching ratio of the invisible decay can reach the level of a few $10 \%$. Decays of the heavier $A / H / H^{ \pm}$bosons, in particular into charginos, neutralinos, sleptons and top squarks, are in turn possible. However, for $\tan \beta \gtrsim 10$, they are strongly suppressed.

The second scenario of interest occurs when SUSY particles contribute to loop-induced Higgs decays. If scalar quarks are relatively light, they can lead to sizable contributions to the decays $h \rightarrow g g$ and $h \rightarrow \gamma \gamma$. Since scalar quarks have Higgs couplings that are not proportional to their masses, their contributions are damped by loop factors $1 / m_{\tilde{Q}}^{2}$ and decouple from the vertices, contrary to the case of the SM quarks. Only when $m_{\tilde{Q}}$ is not too large compared to $M_{h}$, the contributions are significant [51]. This is particularly true for the $\tilde{t}_{1}$ contributions to $h \rightarrow g g$, the reasons being that large $X_{t}$ mixing leads to a $\tilde{t}_{1}$ that is much lighter than all other squarks and that the $h$ coupling to stops involves a component which is proportional to $m_{t} X_{t}$ and, for large $X_{t}$, it can be strongly enhanced. Sbottom mixing, $\propto m_{b} X_{b}$, can also be sizable for large $\tan \beta$ and $\mu$ values and can lead to light $\tilde{b}_{1}$ states with strong couplings to the $h$ boson. In $h \rightarrow \gamma \gamma$ decay, there are in addition 
slepton loops, in particular $\tilde{\tau}$ states which behave like scalar bottom quarks and have a strong mixing at high $\mu \tan \beta$, can make a large impact on the decay rate. Besides, chargino loops also enter the $h \rightarrow \gamma \gamma$ decay mode but their contribution is in general smaller since the Higgs- $\chi \chi$ couplings cannot be strongly enhanced.

For the evaluation of the decay branching ratios of the MSSM Higgs bosons, we use the program HDECAY [59], which incorporates all decay channels including those involving super-particles and the most important sets of higher order corrections and effects.

Coming to Higgs boson production at the LHC, for a SM-like particle $H_{\mathrm{SM}}$ there are essentially four mechanisms for single production [23]. These are $g g$ fusion, $g g \rightarrow H_{\mathrm{SM}}$, vector boson fusion, $q q \rightarrow H_{\mathrm{SM}} q q$, Higgs-strahlung, $q \bar{q} \rightarrow H_{\mathrm{SM}} V$ and $t \bar{t}$ associated Higgs production, $p p \rightarrow t \bar{t} H_{\mathrm{SM}}$. The $g g \rightarrow H_{\mathrm{SM}}$ process proceeds mainly through a heavy top quark loop and is by far the dominant production mechanism at the LHC. For a Higgs boson with a mass of $\approx 126 \mathrm{GeV}$, the cross section is more than one order of magnitude larger than in the other processes. Again for $M_{H_{\mathrm{SM}}} \approx 126 \mathrm{GeV}$, the most efficient detection channels are the clean but rare $H \rightarrow \gamma \gamma$ final states, the modes $H \rightarrow Z Z^{*} \rightarrow 4 \ell^{ \pm}, H \rightarrow$ $W W^{(*)} \rightarrow \ell \ell \nu \nu$ with $\ell=e, \mu$ and, to a lesser extent, also $H_{\mathrm{SM}} \rightarrow \tau^{+} \tau^{-}$. At the LHC and, most importantly, at the Tevatron one is also sensitive to $q \bar{q} \rightarrow H_{\mathrm{SM}}+W / Z \rightarrow b \bar{b}+W / Z$ with $W \rightarrow \ell \nu$ and $Z \rightarrow \ell \ell, \nu \bar{\nu}$.

For the MSSM Higgs bosons, the above situation holds for the $h(H)$ state in the (anti-) decoupling regime. Since $A V V$ couplings are absent, the $A$ boson cannot be produced in Higgs-strahlung and vector boson fusion and the rate for $p p \rightarrow t \bar{t} A$ is strongly suppressed. For the $\Phi=A$ and $h(H)$ states, when we are in the (anti-)decoupling limit, the $b$ quark will play an important role for large $\tan \beta$ values as the $\Phi b b$ couplings are enhanced. One then has to take into account the $b$-loop contribution in the $g g \rightarrow \Phi$ processes which becomes the dominant component in the MSSM and consider associated Higgs production with $b \bar{b}$ final states, $p p \rightarrow b \bar{b}+\Phi$ which become the dominant channel in the MSSM. The latter process is in fact equivalent to $b \bar{b} \rightarrow \Phi$ where the $b$-quarks are taken from the proton in a five active flavor scheme. As the $\Phi$ bosons decay mainly into $b \bar{b}$ and $\tau^{+} \tau^{-}$pairs, with the former being swamped by the QCD background, the most efficient detection channel would be $p p \rightarrow \Phi \rightarrow \tau^{+} \tau^{-}$. This process receives contributions from both the $g g \rightarrow \Phi$ and $b \bar{b} \rightarrow \Phi$ channels.

These processes also dominate the $h / H / A$ production in the intense coupling regime. In fact, in the three regimes above, when all processes leading to $\tau^{+} \tau^{-}$final states are added up, the rate is $2 \times \sigma(g g+b \bar{b} \rightarrow A) \times \operatorname{BR}\left(A \rightarrow \tau^{+} \tau^{-}\right)$. In the intermediate coupling regime, these process have very low cross sections as for $3-5 \leq \tan \beta \leq 7-10$, the $\Phi b b$ couplings are not enough enhanced and the $\Phi t t$ ones that control the $g g$ fusion rate are still suppressed.

Finally, for the charged Higgs boson, the dominant channel is the production from top quark decays, $t \rightarrow H^{+} b$, for masses not too close to $M_{H^{ \pm}}=m_{t}-m_{b}$. This is true in particular at low or large $\tan \beta$ values when the $t \rightarrow H^{+} b$ branching ratio is significant.

The previous discussion on MSSM Higgs production and detection at the LHC might be significantly altered if scalar quarks, in particular $\tilde{t}$ and $\tilde{b}$, are light enough. Indeed, the $H g g$ and $h g g$ vertices in the MSSM are mediated not only by the $t / b$ loops but also by 
loops involving their partners similarly to the Higgs photonic decays. The $g g \rightarrow h$ cross section in the decoupling regime can be significantly altered by light stops and a strong mixing $X_{t}$ which enhances the $h \tilde{t}_{1} \tilde{t}_{1}$ coupling. The cross section times branching ratio $\sigma(g g \rightarrow h) \times \mathrm{BR}(h \rightarrow \gamma \gamma)$ for the lighter $h$ boson at the LHC could be thus different from the SM case, even in the decoupling limit in which the $h$ boson is supposed to be SM-like [51].

Finally, we should note that in the scenario in which the Higgs bosons, and in particular the lightest $h$, decay into invisible lightest neutralinos, $h \rightarrow \chi_{1}^{0} \chi_{1}^{0}$, the observation of the final state will be challenging but possible at the LHC with a higher energy and more statistics. This scenario has recently been discussed in detail in refs. [7, 60].

\section{Analysis and results}

\section{1 pMSSM scans and software tools}

The analysis is based on scans of the multi-parameter MSSM phase space. The input values of the electro-weak parameters, i.e. the top quark pole mass, the $\overline{\mathrm{MS}}$ bottom quark mass, the electro-weak gauge boson masses, electromagnetic and strong coupling constants defined at the scale $M_{Z}$, are given below with their $1 \sigma$ allowed ranges [62],

$$
\begin{aligned}
m_{t} & =(173 \pm 1) \mathrm{GeV}, & \bar{m}_{b}\left(\bar{m}_{b}\right) & =\left(4.19_{-0.06}^{+0.18}\right) \mathrm{GeV}, \\
M_{Z} & =(91.19 \pm 0.002) \mathrm{GeV}, & M_{W} & =(80.42 \pm 0.003) \mathrm{GeV} \\
\alpha\left(M_{Z}^{2}\right) & =1 / 127.916 \pm 0.015, & \alpha_{s}\left(M_{Z}^{2}\right) & =0.1184 \pm 0.0014
\end{aligned}
$$

The pMSSM parameters are varied in an uncorrelated way in flat scans, within the following ranges:

$$
\begin{gathered}
1 \leq \tan \beta \leq 60 \\
50 \mathrm{GeV} \leq M_{A} \leq 3 \mathrm{TeV} \\
-10 \mathrm{TeV} \leq A_{f} \leq 10 \mathrm{TeV} \\
50 \mathrm{GeV} \leq m_{\tilde{f}_{L}}, m_{\tilde{f}_{R}}, M_{3} \leq 3.5 \mathrm{TeV} \\
50 \mathrm{GeV} \leq M_{1}, M_{2},|\mu| \leq 2.5 \mathrm{TeV}
\end{gathered}
$$

to generate a total of $6 \times 10^{7} \mathrm{pMSSM}$ points. The scan range is explicitly chosen to include the various mixing scenarios in the Higgs section discussed in section 2.2: the maximal mixing, no-mixing and typical mixing scenarios. Additional $10^{7}$ points are generated in specialised scans used for the studies discussed later in sections 3.3 and 3.4. We select the set of points fulfilling constraints from flavour physics and lower energy searches at LEP2 and the Tevatron, as discussed in ref. [63], to which we refer also for details on the scans. We highlight here the tools most relevant to this study. The SUSY mass spectra are generated with SuSpect [32] and SOFTSUSY 3.2.3 [33]. The superparticle partial decay widths and branching fractions are computed using the program SDECAY 1.3 [64-67]. The flavour observables and dark matter relic density are calculated with SuperIso Relic v3. $2[68-70]$. 
The Higgs production cross sections at the LHC are computed using HIGLU 1.2 [7173] for the $g g \rightarrow h / H / A$ process, including the exact contributions of the top and bottom quark loops at NLO-QCD and the squark loops, and the program bb@nnlo for $b \bar{b} \rightarrow h / H / A$ at NNLO-QCD. The cross section for $V H$ production is compute at NLO using the V2HV program. These are interfaced with Suspect for the MSSM spectrum and HDECAY for the Higgs decay branching ratios. The Higgs production cross sections and the branching fractions for decays into $b \bar{b}, \gamma \gamma, W W$ and $Z Z$ from HIGLU and HDECAY are compared to those predicted by FeynHiggs. In the SM both the $g g \rightarrow H_{S M}$ cross section and the branching fractions agree within $\sim 3 \%$. Significant differences are observed in the SUSY case, with HDECAY giving values of the branching fractions to $\gamma \gamma$ and $W W, Z Z$ which are on average $9 \%$ lower and $19 \%$ larger than those of FeynHiggs and have an r.m.s. spread of the distribution of the relative difference between the two programs of $18 \%$ and $24 \%$, respectively [7].

\subsection{Constraints}

We apply constraints from flavour physics, anomalous muon magnetic moment, dark matter and SUSY searches at LEP and the Tevatron. These have been discussed in details in ref. [63]. In particular, we consider the decay $B_{s} \rightarrow \mu^{+} \mu^{-}$, which can receive extremely large SUSY contributions at large $\tan \beta$. An excess of events in this channels has been reported by the CDF-II collaboration at the Tevatron [74] and upper limits by the LHCb [75] and CMS [76] collaborations at LHC. Recently the LHCb collaboration has presented their latest result for the search of this decay based on $1 \mathrm{fb}^{-1}$ of data. A $95 \%$ C.L. upper limit on its branching fraction is set at $4.5 \times 10^{-9}$ [75]. After accounting for theoretical uncertainties, estimated at the $11 \%$ level $[77,78]$ the constraint

$$
\operatorname{BR}\left(B_{s} \rightarrow \mu^{+} \mu^{-}\right)<5 \times 10^{-9}
$$

is used in this analysis. For large values of $\tan \beta$, this decay can be enhanced by several orders of magnitude so that strong constraints on the scalar contributions can be derived [79], and the small $M_{A}$ and large $\tan \beta$ region can be severely constrained. As already remarked in ref. [63], the constraints obtained are similar and complementary to those from the dark matter direct detection limits of XENON-100 [80] and searches for the $A \rightarrow \tau^{+} \tau^{-}$decay.

Concerning the relic density constraint, we impose the upper limit derived from the WMAP-7 result [81]

$$
10^{-4}<\Omega_{\chi} h^{2}<0.155
$$

accounting for theoretical and cosmological uncertainties [82, 83].

The searches conducted by the ATLAS and CMS collaborations on the $\sqrt{s}=7 \mathrm{TeV}$ data for channels with missing $E_{T}[84,85]$ have already provided a number of constraints relevant to this study. These have excluded a fraction of the pMSSM phase space corresponding to gluinos below $\sim 600 \mathrm{GeV}$ and scalar quarks of the first two generations below $\sim 400 \mathrm{GeV}$. These constraints are included using the same analysis discussed in ref. [63], extended to an integrated luminosity of $4.6 \mathrm{fb}^{-1}$. In addition, we also include the search conducted by ATLAS for the large missing transverse momentum and two $b$-tagged jets 


\begin{tabular}{|c|c|c|}
\hline Parameter & Value & Experiment \\
\hline$M_{H}$ & $125.9 \pm 2.1 \mathrm{GeV}$ & ATLAS $[1,2]+$ CMS $[3,4]$ \\
$\mu_{\gamma \gamma}$ & $1.71 \pm 0.33$ & ATLAS $[90]+$ CMS $[91]$ \\
$\mu_{Z Z}$ & $0.95 \pm 0.40$ & ATLAS $[92]+$ CMS $[93]$ \\
$\mu_{b \bar{b}}$ & $<1.64(95 \%$ C.L. $)$ & CMS $[94]$ \\
\hline$\mu_{\tau \tau}$ & $<1.06$ (95\% C.L.) & CMS $[95]$ \\
\hline
\end{tabular}

Table 1. Input parameters used for the pMSSM study.

signature on the $7 \mathrm{TeV}$ data [86]. Searches for the MSSM Higgs bosons in the channels $h / H / A \rightarrow \tau^{+} \tau^{-}[87,88]$ have already excluded a significant fraction of the $\left[M_{A}, \tan \beta\right]$ plane at low $M_{A}$ values, $M_{A} \lesssim 200 \mathrm{GeV}$ and $\tan \beta \lesssim 10$, and larger values of $\tan \beta$ for $M_{A} \gtrsim 200 \mathrm{GeV}$. These constraint on the pMSSM parameter space are thus important. The search for light charged Higgs bosons in top decays, $t \rightarrow b H^{+} \rightarrow b \tau \nu$, performed by the ATLAS collaboration [89], sensitive at low $M_{A}$ values, $M_{A} \lesssim 140 \mathrm{GeV}$, corresponding to $M_{H^{ \pm}} \lesssim 160 \mathrm{GeV}$, provides independent constraints.

Following the new boson discovery at the LHC, the lightest Higgs boson in our analysis is restricted to have a mass in the range allowed by the results reported by ATLAS and CMS:

$$
123 \mathrm{GeV} \leq M_{h} \leq 129 \mathrm{GeV}
$$

where the range is centred around the value corresponding to the average of the Higgs mass values reported by ATLAS and CMS, $M_{h} \simeq 126 \mathrm{GeV}$, with the lower and upper limits accounting for the parametric uncertainties from the SM inputs given in eq. (3.1), in particular the top quark mass, and the theoretical uncertainties in the determination of the $h$ boson mass. It is also consistent with the experimental exclusion bounds.

The impact of the Higgs mass value and its decay rates on the parameters of the pMSSM can be estimated by studying the compatibility of the generated pMSSM points with the preliminary results reported by ATLAS [1, 2] and CMS [3, 4] at the LHC and also by the Tevatron experiments [5]. Starting from our set of $6 \times 10^{7}$ pMSSM points which are pre-selected for compatibility with the constraints discussed above, we consider the two decay channels giving the new boson evidence at the LHC, $\gamma \gamma$ and $Z Z$, and include also the $b \bar{b}$ and $\tau \tau$ channels. In the following, we use the notation $R_{X X}$ to indicate the Higgs decay branching fraction to the final state $X X, \mathrm{BR}(h \rightarrow X X)$, normalised to its SM value. We also compute the ratios of the product of production cross sections times branching ratios for the pMSSM points to the SM values, denoted by $\mu_{X X}$ for a given $h \rightarrow X X$ final state, $\mu_{X X}=\frac{\sigma(h) \times \mathrm{BR}(h \rightarrow X X)}{\sigma\left(H_{\mathrm{SM}}\right) \times \mathrm{BR}\left(H_{\mathrm{SM}} \rightarrow X X\right)}$, where $\sigma(h)$ is the relevant production cross section. These are compared to the experimental values. For the $\gamma \gamma$ and $Z Z$ channels we take weighted averages of the results just reported by the experiments, as given in table 1 with their estimated uncertainties. 
While the results are compatible with the SM expectations within the present accuracy, they highlight a possible enhancement in the observed rates for the $\gamma \gamma$ channel, where ATLAS and CMS obtain $\mu_{\gamma \gamma}=1.9 \pm 0.5$ and $1.56 \pm 0.43$, respectively. In the following, we do not take into account the theoretical uncertainties in the production cross section, which are estimated significant for the main production channel, $g g \rightarrow h[96,97]$.

\subsection{The decoupling regime}

First we study the mass constraints. Figure 2 presents the parameter space $\left[M_{A}, \tan \beta\right]$ in our benchmark scenario with $M_{S}=2 \mathrm{TeV}$ in the maximal mixing scenario. The regions excluded by the various constraints that we have imposed are indicated. The green area corresponds to the non-observation of Higgs bosons at LEP2 which excludes $\tan \beta \lesssim 3$ at moderate to large $M_{A}$ values, $M_{A} \gtrsim 150 \mathrm{GeV}$, but up to $\tan \beta \approx 5-10$ at low $M_{A}$ values. The blue area is the one ruled out by the latest published results of the CMS collaboration on the search of resonances decaying into $\tau^{+} \tau^{-}$final states; it touches the LEP2 band at small $M_{A}$, but reduces in size when $M_{A}$ is increased. The small visible area in red is the one excluded by the $B_{s} \rightarrow \mu^{+} \mu^{-}$constraint but, in fact, part of the excluded region is hidden by the CMS blue area.

To that, we superimpose the area in which we make the requirement $123 \leq M_{H} \leq$ $129 \mathrm{GeV}$, that is indicated in dark blue. This band covers the entire range of $M_{A}$ values and leaves only the $\tan \beta$ values that are comprised between $\tan \beta \approx 3-5$ and $\tan \beta \approx 10$. Between the LEP2 and the " $M_{h}$ " blue band, one has $M_{h}<123 \mathrm{GeV}$, while above the $M_{h}$ band, one has $M_{h}>129 \mathrm{GeV}$ and both areas are excluded. The requirement that the $h$ boson mass should have the value measured at the LHC, even with the large uncertainty that we assume, provides thus a strong constraint on the $\left[M_{A}, \tan \beta\right]$ parameter space in the pMSSM.

In figure 3 , we show the same $\left[M_{A}, \tan \beta\right]$ plane but for different SUSY-breaking scales, $M_{S}=1,2$ and $3 \mathrm{TeV}$ and for the zero, typical and maximal mixing scenarios defined in eqs. (4-6). As can be seen, the situation changes dramatically depending on the chosen scenario. Still, in the maximal mixing scenario with $M_{S}=3 \mathrm{TeV}$ the size of the $M_{h}$ band is reduced from above, as in this case, already values $\tan \beta \gtrsim 5$ leads to a too heavy $h$ boson, $M_{h} \gtrsim 129 \mathrm{GeV}$. In turn, for $M_{S}=1 \mathrm{TeV}$, the entire space left by the LEP 2 and CMS Higgs constraints is covered with many points at $\tan \beta \gtrsim 20$ excluded by the flavor constraint. Nevertheless, the scenario with $M_{S} \approx 1 \mathrm{TeV}$ will be challenged by the search for squarks at the LHC when $30 \mathrm{fb}^{-1}$ of data will be collected by the experiments. In the no-mixing scenario, it is extremely hard to obtain a Higgs mass of $M_{h} \geq 123 \mathrm{GeV}$ and all parameters need to be maximised: $M_{S}=3 \mathrm{TeV}$ and $\tan \beta \gtrsim 20$; a small triangle is thus left over, the top of which is challenged by the flavor constraints. The typical mixing scenario resembles the no-mixing scenario, with the notable difference that for $M_{S}=3 \mathrm{TeV}$, the entire space, not excluded by the LEP2 and CMS constraints, provides us with an acceptable value of $M_{h}$.

In the discussion so far, we have adopted the value $m_{t}=(173 \pm 1) \mathrm{GeV}$ for the top quark mass as measured by the CDF and D0 experiments at the Tevatron [98]. This implicitly assumes that this mass corresponds to the top quark pole mass, i.e. the mass in the on-shell scheme, which serves as input in the calculation of the radiative corrections in 


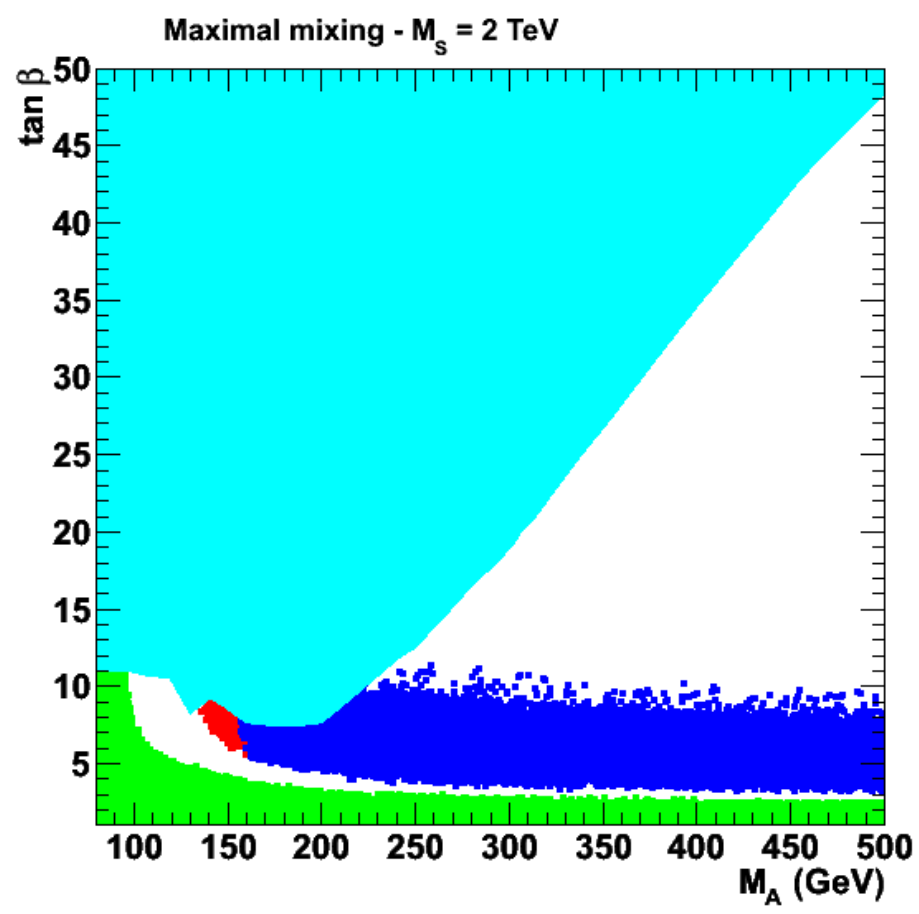

Figure 2. The parameter space $\left[M_{A}, \tan \beta\right]$ for $M_{S}=2 \mathrm{TeV}$ in the maximal mixing scenario with the individual constraints from LEP2 (green), CMS $\tau^{+} \tau^{-}$searches (light blue) and flavor physics (red) displayed. The area in which $123 \leq M_{H} \leq 129 \mathrm{GeV}$ is also shown (dark blue).

the pMSSM Higgs sector and, in particular, to the mass $M_{h}$. However, the mass measured at the Tevatron is not theoretically well defined and it is not proved that it corresponds indeed to the pole mass as discussed in [99]. For an unambiguous and well-defined determination of the top quark mass, it is appears to be safer to use the value obtained from the determination of the top quark pair production cross section measured at the Tevatron, by comparing the measured value with the theoretical prediction at higher orders. This determination has been recently performed yielding the value of $(173.3 \pm 2.8) \mathrm{GeV}$ [99] for $m_{t}^{\text {pole }}$. The central value is very close to that measured from the event kinematics but its uncertainty is larger, as a result of the experimental and theoretical uncertainties affecting this measurement.

Therefore, it is interesting to assess the impact of a broader mass range for the top quark. We return to our benchmark scenario with $M_{s}=2 \mathrm{TeV}$ and maximal stop mixing and draw the " $M_{h}$ " bands using the top quark mass values of $170 \mathrm{GeV}$ and $176 \mathrm{GeV}$ corresponding to the wider uncertainty interval quoted above. The result is shown in figure 4. A $1 \mathrm{GeV}$ change in $m_{t}$ input leads to a $\sim 1 \mathrm{GeV}$ change in the corresponding $M_{h}$ value. The smaller value of $m_{t}$ would open up more parameter space as the region in which $M_{h} \gtrsim 129 \mathrm{GeV}$ will be significantly reduced. In turn, for $m_{t}=176 \mathrm{GeV}$, the corresponding $h$ boson mass increases and the dark-blue area quite significantly shrinks, as a result. It must be noted that for $m_{t}=170 \mathrm{GeV}$, the no-mixing scenario would be totally excluded for $M_{S} \lesssim 3 \mathrm{TeV}$, while in the typical mixing scenario only a small area at high $\tan \beta$ will 

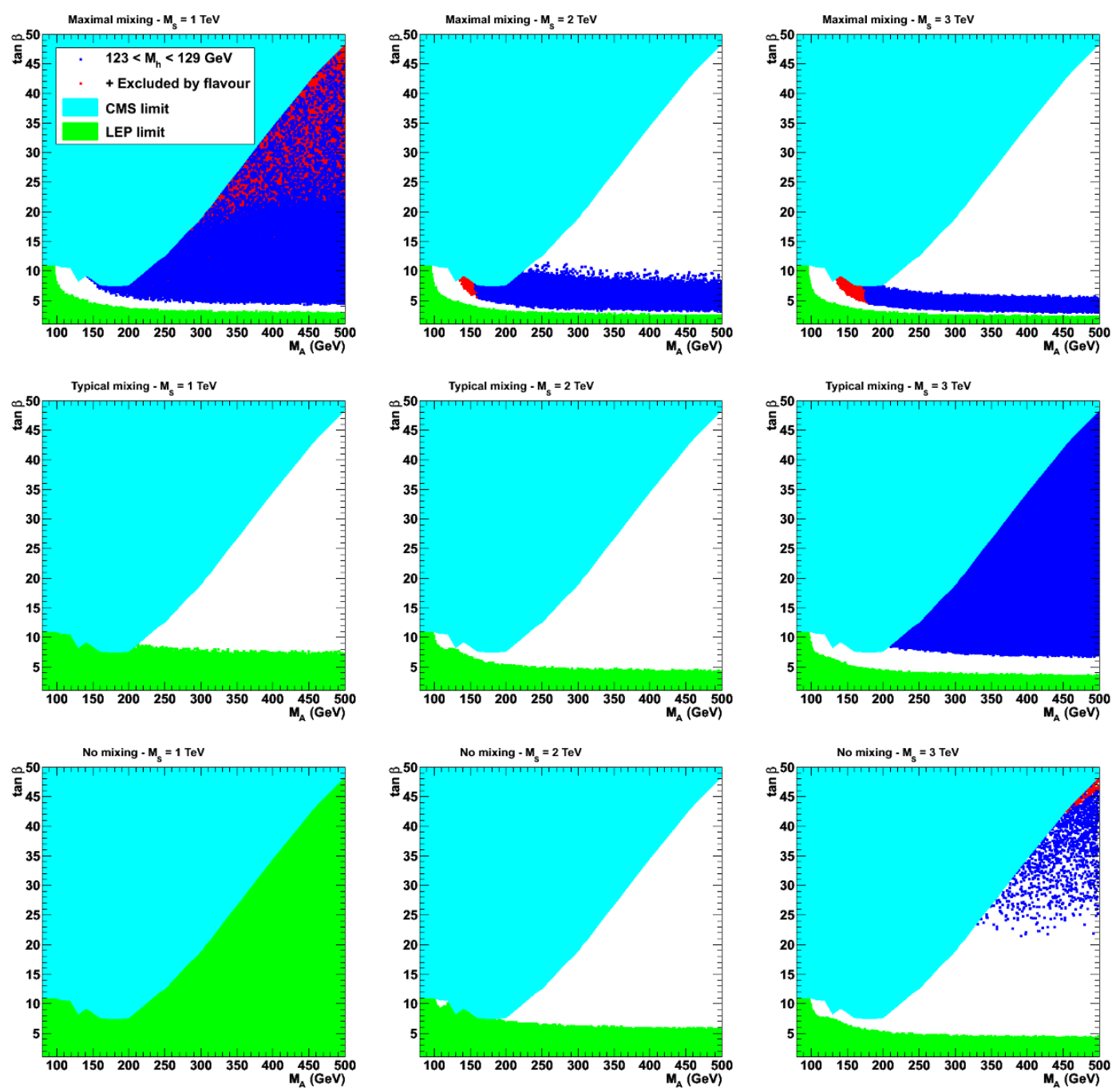

Figure 3. The $\left[M_{A}, \tan \beta\right]$ plane for $M_{S}=1,2$ and $3 \mathrm{TeV}$ and for zero, typical and maximal mixing. The colour coding for the different regions is the same as in figure 2 .

remain viable. For $m_{t}=176 \mathrm{GeV}$, significantly broader $\left[M_{A}, \tan \beta\right]$ regions, excluded when taking the $\pm 1 \mathrm{GeV}$ uncertainty for top mass value, become allowed. The impact of the value of $m_{t}$ is thus extremely significant. This is even more true in constrained scenarios, where the top mass also enters in the evaluation of the soft SUSY-breaking parameters and the minimisation of the scalar potential. To visualise the impact of $m_{t}$, we have repeated the study presented in ref. [6], presenting the maximal $M_{h}$ value reached when scanning over all the parameters of the minimal SUGRA, AMSB and GMSB models. Figure 5 shows the result with the $M_{h}^{\max }$ value as a function of $M_{S}$ taking $m_{t}=173 \pm 3 \mathrm{GeV}$. While for $m_{t}$ $=173 \mathrm{GeV}$, there is no region of the parameter space of the mAMSB and mGMSB models which satisfies $M_{h} \gtrsim 123 \mathrm{GeV}$, for $M_{S} \lesssim 3 \mathrm{TeV}$ assumed in [6], and the models are disfavoured, using $m_{t}=176 \mathrm{GeV}$, the regions of these mAMSB and mGMSB models beyond $M_{S}=2 \mathrm{TeV}$ become again viable. This will be also the case of some of the variants and 

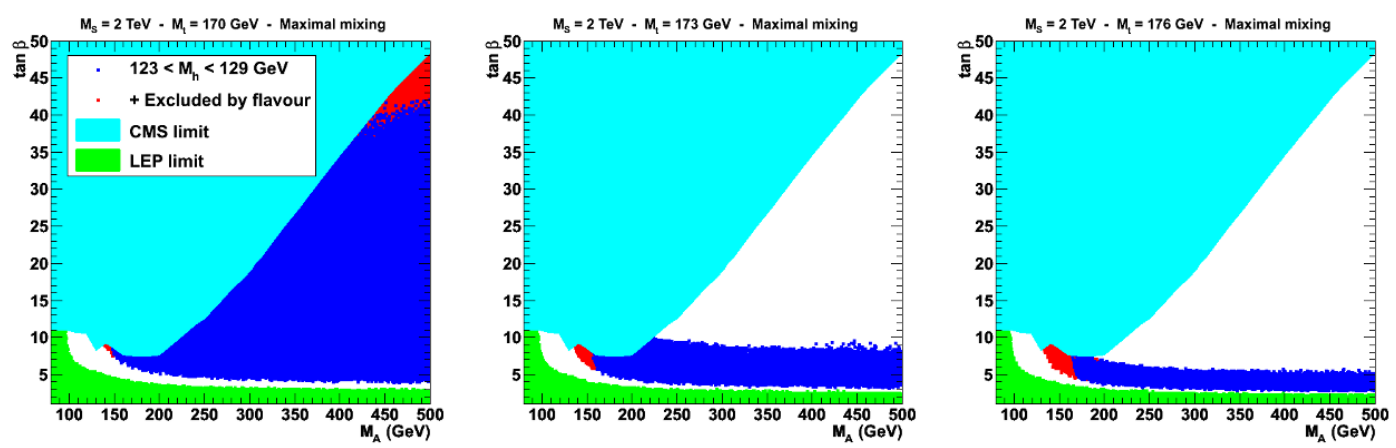

Figure 4. The parameter space $\left[M_{A}, \tan \beta\right]$ for $M_{S}=2 \mathrm{TeV}$, maximal mixing and three values of the top quark mass $m_{t}=170 \mathrm{GeV}$ (left), $173 \mathrm{GeV}$ (centre) and $176 \mathrm{GeV}$ (right).

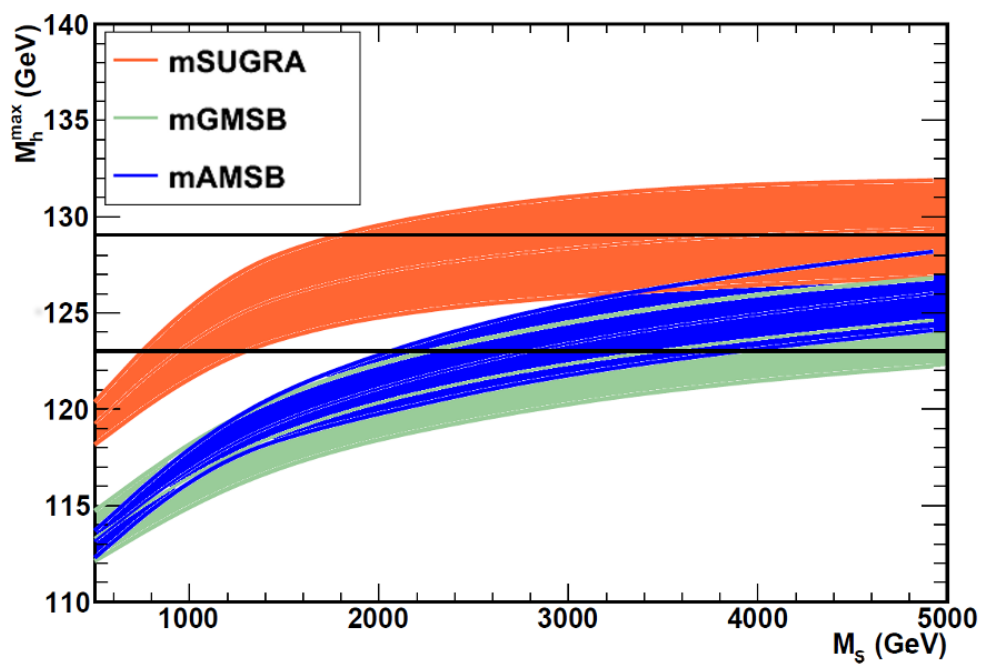

Figure 5. Maximal Higgs mass in the constrained MSSM scenarios mSUGRA, mAMSB and mGMSB, an a function of the scale $M_{S}$ when the top quark mass is varied in the range $m_{t}=170$ $176 \mathrm{GeV}$.

even more constrained mSUGRA scenarios. Further, even for $m_{t}=173 \mathrm{GeV}$, if we move the $M_{S}$ upper limit from the $3 \mathrm{TeV}$ boundary adopted in ref. [6] to $M_{S}=5 \mathrm{TeV}$, these models have region of their parameters compatible with the LHC Higgs mass range.

Finally, we comment on the impact of increasing the $M_{h}$ allowed range from $123 \mathrm{GeV}$ $\leq M_{h} \leq 127 \mathrm{GeV}$ as was done in ref. [6] relying on the 2011 LHC data, to that adopted here, $123 \mathrm{GeV} \leq M_{h} \leq 129 \mathrm{GeV}$, in the various constrained models discussed there, to which we also refer for the definition of the models and the ranges of input parameters adopted. The outcome is shown in figure 6 , where the maximal $h$ mass value obtained by scanning the input parameters of the models over their appropriate ranges, are given as a function of $\tan \beta$ and $M_{S}$. As the lower bound $M_{h}^{\max } \geq 123 \mathrm{GeV}$ is the same as in our earlier analysis, the mASMB, mGMSB and some variants of the mSUGRA model such as the constrained NMSSM (cNMSSM), the no-scale model and the very constrained MSSM (VCMSSM) scenarios are still disfavoured. However, for mSUGRA and the non-universal 

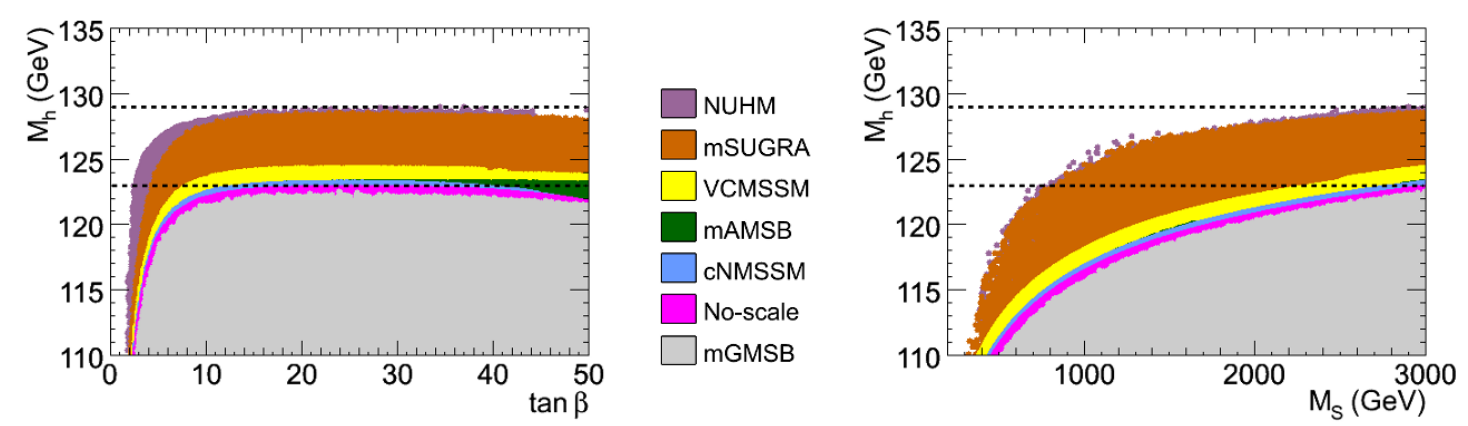

Figure 6. The maximal $h$ mass value $M_{h}^{\max }$ as functions of $\tan \beta$ (left) and $M_{S}$ (right) in the mASMB, mGMSB as well as in mSUGRA and some of its variants. The basic parameters of the models are varied within the ranges given in ref. [6]; the top quark mass is fixed to $m_{t}=173 \mathrm{GeV}$.

Higgs mass model (NUHM), all values of $\tan \beta \gtrsim 3$ and $1 \mathrm{TeV} \lesssim M_{S} \lesssim 3 \mathrm{TeV}$ lead to an appropriate value of $M_{h}$ when including the uncertainty band.

\subsection{The other (non-SUSY) regimes}

The other regimes of the pMSSM Higgs sector, apart from the decoupling and the SUSY regimes, occur for low to intermediate values of the pseudoscalar Higgs mass, $M_{A} \lesssim 200 \mathrm{GeV}$, and relatively large $\tan \beta$ values. These are the anti-decoupling, the intense, the intermediate and the vanishing coupling regimes. They are constrained by the results of the LEP2 and LHC searches. The LEP2 results for $M_{A} \lesssim 200 \mathrm{GeV}$ and not too large $M_{S}$ values, lead to $\tan \beta \gtrsim 3,8$ and 10 for, respectively the maximal, typical and no-mixing scenarios; see figure 3 . The negative search for Higgs particles in $\tau$-lepton final states, $p p \rightarrow \Phi \rightarrow \tau^{+} \tau^{-}$, by the ATLAS and CMS collaborations places further constraints. While in the decoupling regime, the relevant Higgs states would be $\Phi=A+H$, these are $\Phi=A+h$ and $\Phi=A+H+h$ in the anti-decoupling and intense coupling regimes, respectively. As already mentioned, one would have in the three regimes the same signal cross section times branching ratios $\sigma\left(p p \rightarrow \Phi \rightarrow \tau^{+} \tau^{-}\right) \approx \sigma(b \bar{b}+g g \rightarrow A) \times \mathrm{BR}\left(A \rightarrow \tau^{+} \tau^{-}\right)$ almost independently of the mixing scenario and the other pMSSM parameters [97]. The constraint from the CMS published results alone with the $\approx 5 \mathrm{fb}^{-1}$ of data collected in 2011 [88] imposes $\tan \beta \gtrsim 10$, as shown in figure 7 where we zoom on the plane $\left[M_{A}, \tan \beta\right]$ at low to intermediate $M_{A}$ values, for the maximal mixing scenario and $M_{S}=2 \mathrm{TeV}$.

This limit can be strengthened by the same $\tau^{+} \tau^{-}$search performed by the ATLAS collaboration [87] and also by the $t \rightarrow H^{+} b$ search in top decays [89] which is effective for $M_{A}=\sqrt{M_{H^{ \pm}}^{2}+M_{W}^{2}} \lesssim 130 \mathrm{GeV}$ and which, as can be seen in figure 7, excludes the large $\tan \beta$ values for which $\mathrm{BR}\left(t \rightarrow b H^{+}\right)$is significant. Put together, these constraints exclude entirely both the anti-decoupling and intense coupling regimes. The intermediate coupling regime with $\tan \beta \approx 5-8$ remains, also when the LEP2 constraint is imposed. Depending on the mixing scenario, most of this is excluded by the $M_{h} \approx 126 \mathrm{GeV}$ constraint (see figure 3).

A very interesting possibility would be that the observed Higgs particle at the LHC is actually the $H$ state, while the lighter $h$ boson has suppressed couplings to $W / Z$ bosons and 


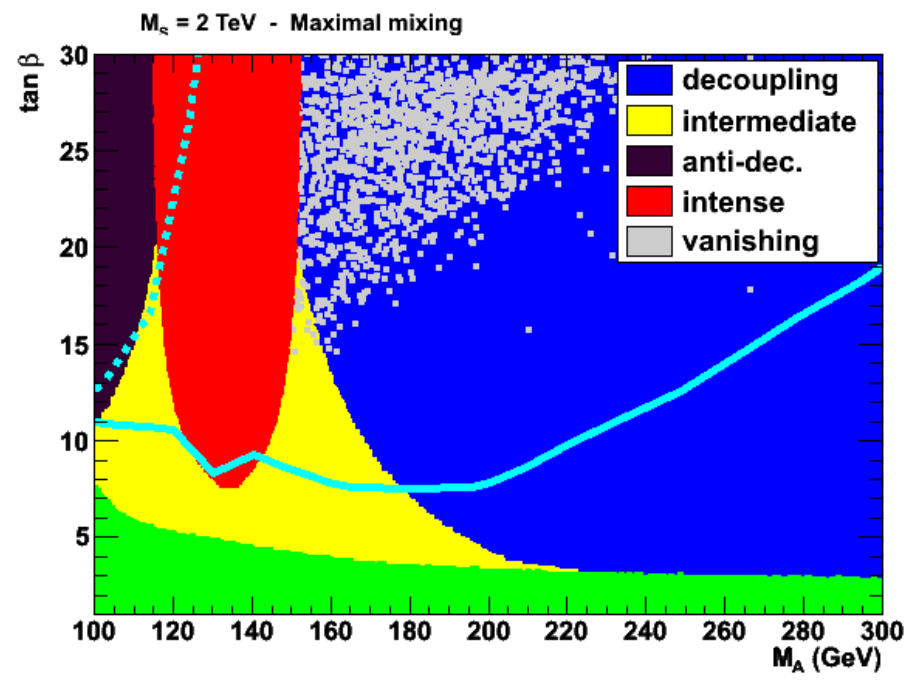

Figure 7. Parameter space for the various regimes of the MSSM Higgs sector as defined in the text and in eq. (8) in the $\left[M_{A}, \tan \beta\right]$ plane, in the maximal mixing scenario with $M_{S}=2 \mathrm{TeV}$. The upper limit constraints from $\Phi \rightarrow \tau \tau$ (continuous light blue line) and $t \rightarrow H^{+} b$ (dashed blue line) searches at the LHC are shown together with the LEP2 excluded region (green area).

top quarks, allowing it to escape detection. In this case, the $H$ couplings to bottom quarks should not be too enhanced, $\tan \beta \lesssim 8$, not to be in conflict with the $\tau^{+} \tau^{-}$and $t \rightarrow b H^{+}$ searches above. For the $H$ boson to be SM-like, one should have $M_{A} \approx M_{H} \approx 126 \mathrm{GeV}$ and not too low $\tan \beta$ values, $\tan \beta \gtrsim 7-10$. One is then in the borderline between the anti-decoupling and the intermediate coupling regimes. We have searched for points in which indeed $M_{H} \approx 126 \mathrm{GeV}$ with couplings to $V V$ states, $g_{H V V} \gtrsim 0.9$, such that the $H \rightarrow Z Z$ and $H \rightarrow \gamma \gamma$ (which mainly occurs through a $W$-boson loop) decays are not suppressed compared to the measured values by ATLAS and CMS given in table 1 . In our scan, out of the $10^{6}$ points, before imposing any LHC-Higgs constraint, only $\approx 20$ points fulfilled the above requirements. These points are then completely excluded once the flavor constraints, in particular those from the $b \rightarrow s \gamma$ radiative decay, are imposed. Hence, the possibility that the observed Higgs particle at the LHC is not the lightest $h$ particle appears highly unlikely according to the result of our scan of the parameter space. Combining the $h / A \rightarrow \tau^{+} \tau^{-}$and the $t \rightarrow b H^{+}$constraints and including the results on the new $8 \mathrm{TeV}$ data should further constrain the parameter space and completely exclude this scenario.

Finally, the vanishing coupling regime is strongly disfavoured by the LHC and Tevatron data that are summarised in table 1 . The observation of $H \rightarrow Z Z$ final states by the ATLAS and CMS collaborations rules out the possibility of vanishing $h V V$ couplings. The reported excess of events in the $q \bar{q} \rightarrow V H \rightarrow V b \bar{b}$ process by the CDF and D0 collaborations would rule out both the vanishing $h b b$ and $h V V$ coupling scenarios, if confirmed. However, these couplings may still be smaller than those predicted in the SM, in particular due to the effects of SUSY particles at high $\tan \beta$ [45-50]. We are then in the SUSY-regime to which we turn now. 


\subsection{The SUSY regime}

In the SUSY regime, both the Higgs production cross section in gluon-gluon fusion and the Higgs decay rates can be affected by the contributions of SUSY particles. This makes a detailed study of the pMSSM parameter space in relation to the first results reported by the ATLAS and CMS collaborations especially interesting for its sensitivity to specific regions of the pMSSM parameter space. In particular, the branching fraction for the $\gamma \gamma$ decay of the $h$ state is modified by Higgs mixing effects outside the decoupling regime as was discussed above, by a change of the $h b b$ coupling due to SUSY loops [45-50], by light superparticle contributions to the $h \gamma \gamma$ vertex $[8,51,52]$ and by invisible $h$ decays into light neutralinos [54-58].

We study these effects on the points of our pMSSM scan imposing the LHC results as constraints. The numerical values adopted in the analysis are given in table 1 , assuming in the following on that the observed particle is the $h$ state. First, we briefly summarise the impact of the SUSY particles on the Higgs decay branching fractions, staring from invisible decays, and production cross sections. Then we discuss our finding on the impact of the LHC and Tevatron data on the pMSSM parameters.

\subsubsection{Invisible Higgs decays}

Despite the fact that the discovered particle has a sufficiently large event rate in visible channels to achieve a $5 \sigma$ observation, it is interesting to consider the regions of parameter space in which invisible Higgs may decays occur. This scenario has recently been reconsidered in $[7,60]$. Besides the value of $M_{h}$, the invisible branching ratio $\mathrm{BR}\left(h \rightarrow \chi_{1}^{0} \chi_{1}^{0}\right)$ is controlled by four parameters: the gaugino masses $M_{1}$ and $M_{2}$, the higgsino parameter $\mu$ and $\tan \beta$. They enter the $4 \times 4$ matrix $Z$ which diagonalises the neutralino mass matrix. They also enter the Higgs coupling to neutralinos which, in the case of the LSP, is

$$
g_{h \chi_{1}^{0} \chi_{10}} \propto\left(Z_{12}-\tan \theta_{W} Z_{11}\right)\left(\sin \beta Z_{13}+\cos \beta Z_{14}\right)
$$

if we assume the decoupling limit not to enhance the $h \rightarrow b \bar{b}$ channel which would significantly reduce the invisible decay. In this coupling, $Z_{11}, Z_{12}$ are the gaugino components and $Z_{13}, Z_{14}$ the higgsino components. Thus, the coupling vanishes if the LSP is a pure gaugino, $|\mu| \gg M_{1}$ leading to $m_{\chi_{1}^{0}} \approx M_{1}$, or a pure higgsino, $M_{1} \gg|\mu|$ with $m_{\chi_{1}^{0}} \approx|\mu|$.

For the invisible decay to occur, a light LSP, $m_{\chi_{1}^{0}} \leq \frac{1}{2} M_{h}$ is required. Since in the pMSSM, the gaugino mass universality $M_{2} \approx 2 M_{1}$ is relaxed, one can thus have a light neutralino without being in conflict with data. The constraint from the $Z$ invisible decay width measured at LEP restricts the parameter space to points where the $\tilde{\chi}_{1}^{0}$ is bino-like, if its mass is below $45 \mathrm{GeV}$, and thus to relatively large values of the higgsino mass parameter $|\mu|$. Since a large decay width into $\tilde{\chi}_{1}^{0} \tilde{\chi}_{1}^{0}$ corresponds to small values of $|\mu|$, this remove a large part of the parameter space where the invisible Higgs decay width is sizable. Still, we observe invisible decays for $45 \mathrm{GeV}<M_{\tilde{\chi}^{0}}<M_{h^{0}} / 2$ and $|\mu|<150 \mathrm{GeV}$, corresponding to a combination of parameters where the $\tilde{\chi}_{1}^{0}$ is a mixed higgsino-gaugino state [7]. These pMSSM points are shown in the $\left[M_{1}, \mu\right]$ plane in the left panel of figure 8 .

If the LSP at such a low mass were to be the dark-matter particle, with the relic density given in eq. (12), it should have an efficient annihilation rate into SM particles. 

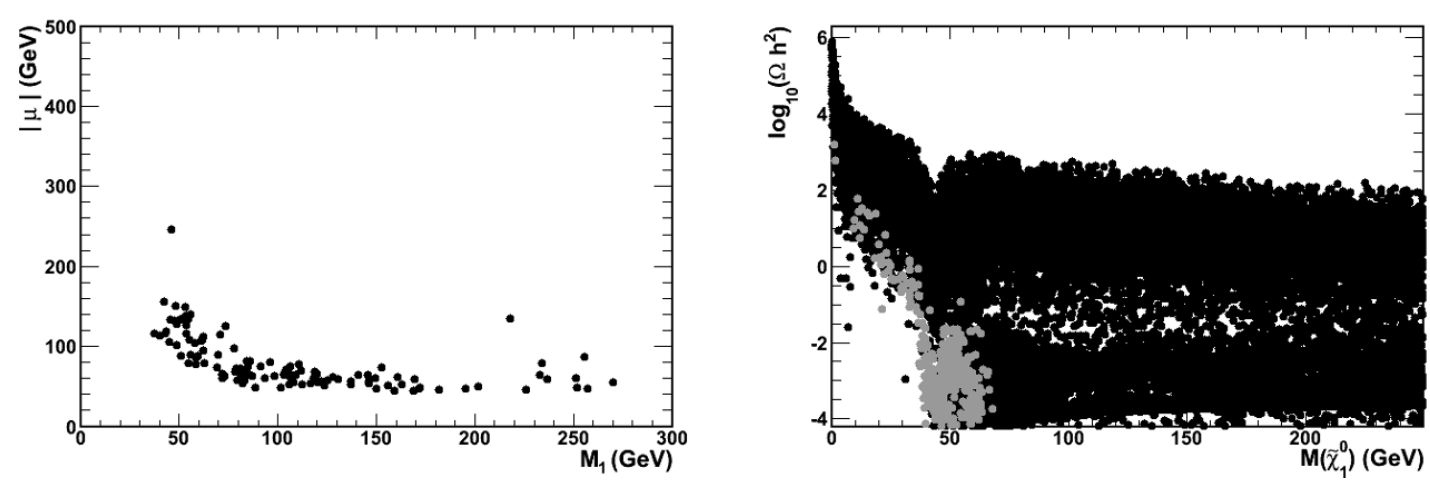

Figure 8. Left: points in the $\left[M_{1}, \mu\right]$ parameter space where the invisible branching fraction $\mathrm{BR}\left(h \rightarrow \chi_{1}^{0} \chi_{1}^{0}\right) \geq 0.15$ from a pMSSM scan where we impose the LEP constraint on the $Z$ invisible width and neutralino relic density $\Omega_{\chi} h^{2}$. Right: $\Omega_{\chi} h^{2}$ as a function of $m_{\chi_{1}^{0}}$ with all the selected pMSSM points in black and those giving a $\operatorname{BR}\left(h \rightarrow \chi_{1}^{0} \chi_{1}^{0}\right) \geq 0.15$ in grey.

The only possible way for that to occur would be $\chi_{1}^{0} \chi_{1}^{0}$ annihilation through the $s$-channel light $h$ pole $^{3}[100-102]$ which implies that $m_{\chi_{1}^{0}} \lesssim \frac{1}{2} M_{h}$ to still have a non-zero invisible branching ratio, as shown in the right panel of figure 8, where the pMSSM points satisfying $\operatorname{BR}\left(h \rightarrow \chi_{1}^{0} \chi_{1}^{0}\right) \geq 5 \%$ are shown in the plane $\left[m_{\chi_{1}^{0}}, \log _{10}\left(\Omega h^{2}\right)\right]$. However, because the partial decay width $\Gamma\left(h \rightarrow \chi_{1}^{0} \chi_{1}^{0}\right)$ is suppressed by a factor $\beta^{3}$ near the $M_{h} \approx 2 m_{\chi_{1}^{0}}$ threshold, with the velocity $\beta=\left(1-4 m_{\chi_{1}^{0}}^{2} / M_{h}^{2}\right)^{1 / 2}$, the invisible branching fraction is rather small if the WMAP dark matter constraint is to hold. MSSM light neutralinos compatible with claims of direct detection dark matter signals are also consistent with collider bounds [103].

\subsubsection{Sparticle effects on the hb̄ coupling}

SUSY particles will contribute to the $h b \bar{b}$ coupling as there are additional one-loop vertex corrections that modify the tree-level Lagrangian that incorporates them [45-50]. These corrections involve bottom squarks and gluinos in the loops, but there are also possibly large corrections from stop and chargino loops. Both can be large since they grow as $\mu \tan \beta$ or $A_{t} \mu \tan \beta[45-50]$

$$
\Delta_{b} \approx \frac{2 \alpha_{s}}{3 \pi} \frac{m_{\tilde{g}} \mu \tan \beta}{\max \left(m_{\tilde{g}}^{2}, m_{\tilde{b}_{1}}^{2}, m_{\tilde{b}_{2}}^{2}\right)}+\frac{m_{t}^{2}}{8 \pi^{2} v^{2} \sin ^{2} \beta} \frac{A_{t} \mu \tan \beta}{\max \left(\mu^{2}, m_{\tilde{t}_{1}}^{2}, m_{\tilde{t}_{2}}^{2}\right)} .
$$

Outside the decoupling limit, the reduced $b \bar{b}$ couplings of the $h$ state are given in this case by

$$
g_{h b b} \approx g_{A b b} \approx \tan \beta\left(1-\Delta_{b}\right)
$$

\footnotetext{
${ }^{3}$ The other possible channels are strongly suppressed or ruled out. The co-annihilation with charginos, heavier neutralinos and staus is not effective as these particles need to be heavier than $\approx 100 \mathrm{GeV}$ and thus the mass difference with the LSP is too large. The annihilation through the $A$-pole needs $M_{A} \approx 2 m_{\chi_{1}^{0}} \lesssim M_{h}$ and sizable $\tan \beta$ values, which is the anti-decoupling regime that is excluded as discussed above. Remains then the bulk region with staus exchanged in the $t$-channel in $\chi_{1}^{0} \chi_{1}^{0} \rightarrow \tau^{+} \tau^{-}$(sbottoms are too heavy) which is difficult to enhance as the LSP is bino-like.
} 

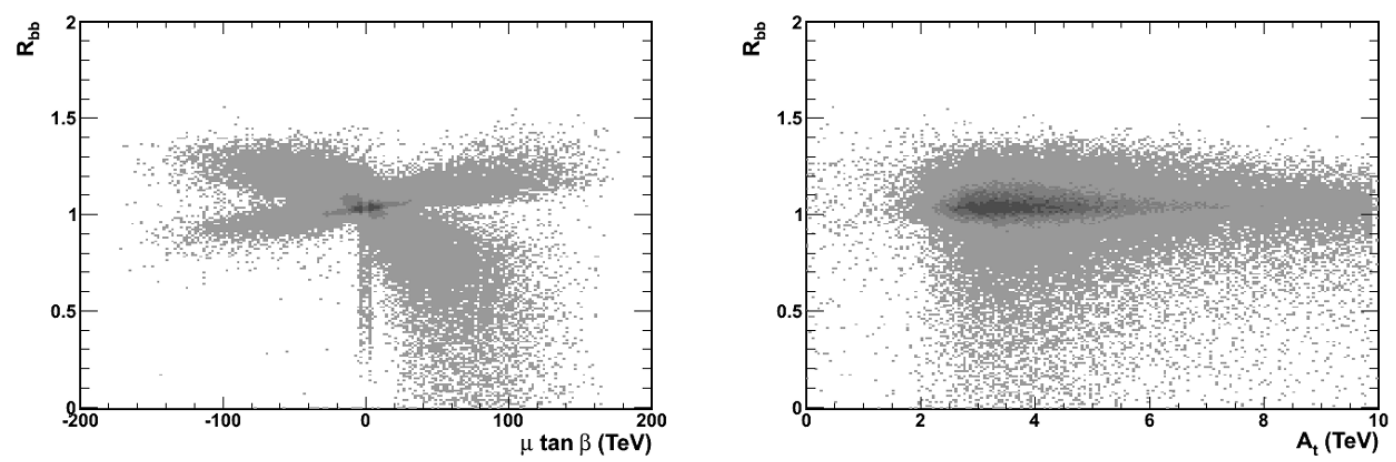

Figure 9. (Left) $R_{b b}$ values for a sample of pMSSM points as a function of the product of the $\mu \tan \beta$ showing the reduction at large values of $\mu \tan \beta$. The reduction in a narrow strip at small values of $\mu$ is due to decays into $\chi \chi$. (Right) the same as a function of $A_{t}$.
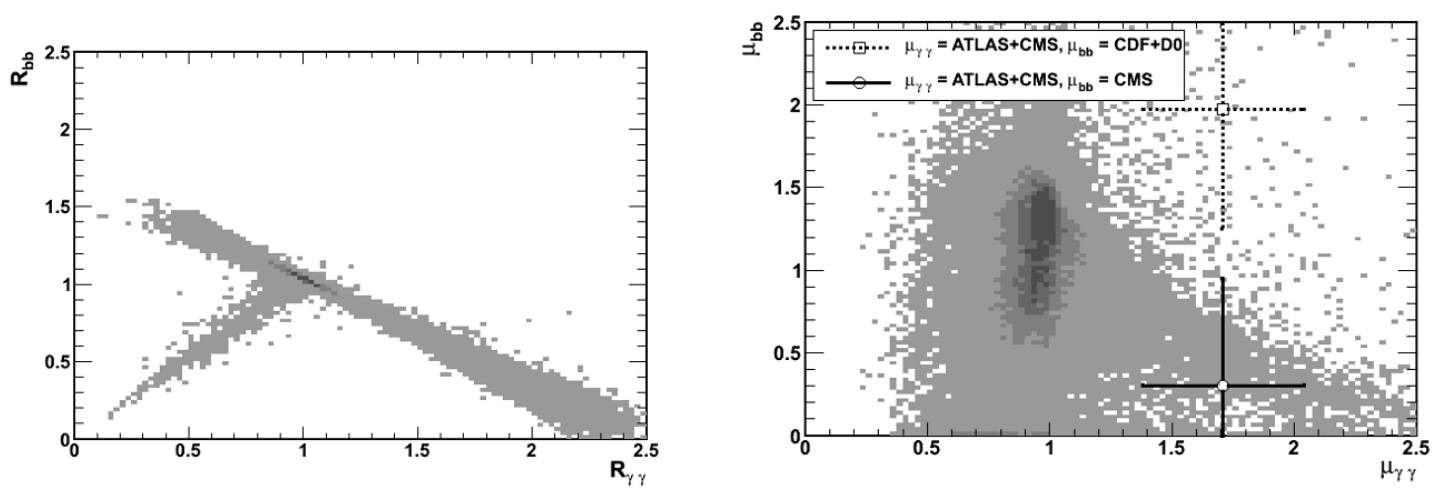

Figure 10. (Left) $R_{b b}$ as a function of $R_{\gamma \gamma}$, showing their anti-correlated variation; the points corresponding to a decrease of both ratios are due to an enhancement of invisible decays to light neutralinos. (Right) $\mu_{b b}$ as a function of $\mu_{\gamma \gamma}$, the anti-correlation is still visible in the region of large $\gamma \gamma$ rate. The values of $\mu_{\gamma \gamma}$ obtained by ATLAS+CMS and $\mu_{b b}$ corresponding to the CMS and $\mathrm{CDF}+\mathrm{D} 0$ searches are overlayed for comparison.

and can be thus significantly reduced or enhanced ${ }^{4}$ depending on the sign of $\mu$ and, possibly, also $A_{t}$. This is exemplified in the left panel of figure 9 , where the ratio $R_{b b} \equiv \operatorname{BR}(h \rightarrow$ $b \bar{b}) / \mathrm{BR}\left(H_{\mathrm{SM}} \rightarrow b \bar{b}\right)$ is shown as a function of the parameter $\mu \tan \beta$ before the constraints of table 1. The two branches in the histogram are due to the sbottom and stop contributions in which $R_{b b}$ is increased or decreased depending on the sign of $\mu$.

A deviation of the partial $h \rightarrow b \bar{b}$ width will enter the total Higgs width, which is dominated by the $b \bar{b}$ channel, and change the $R$ values for the different Higgs decay channels. A reduction of $R_{b b}$ would thus lead to an enhancement of the $\gamma \gamma$ and the $W W / Z Z$ branching fractions compared to their SM values. Figure 10 shows the correlation between the $b b$ and $\gamma \gamma$ decays. The values of $R_{b b}$ vs. $R_{\gamma \gamma}$ bar shown in the left panel, where we

\footnotetext{
${ }^{4}$ These corrections also affect the Higgs production cross sections in the channels $g g+b \bar{b} \rightarrow \Phi$. However, in the cross sections times branching ratios for the $\tau^{+} \tau^{-}$final states, they almost entirely cancel as they appear in both the production rate and the total Higgs decay width [97].
} 
observe a highly anti-correlated variation of the two ratios of branching fraction, $R$, with the exception of the cases where the opening of the decay $h \rightarrow \chi \chi$ suppresses the rates in both channels. These features are still present when considering the $\mu$ products with the relevant production cross sections, $g g \rightarrow h$ for $\gamma \gamma$ and $V H$ for $b \bar{b}$, as shown in the right panel of figure 10, with the preliminary results from LHC and the Tevatron overlayed for comparison.

\subsubsection{Sparticle contributions to the hgg and h $\gamma \gamma$ vertices}

Scalar top quarks can alter significantly the $g g \rightarrow h$ cross section as well as the $h \rightarrow \gamma \gamma$ decay width [51]. The current eigenstates $\tilde{t}_{L}, \tilde{t}_{R}$ mix strongly, with a mixing angle $\propto m_{t} X_{t}$, so that for large $X_{t}=A_{t}-\mu / \tan \beta$ values $^{5}$ there is a lighter mass eigenstate $\tilde{t}_{1}$ which can be much lighter than all other scalar quarks, $m_{\tilde{t}_{1}} \ll M_{S}$. The coupling of the $h$ boson to the $\tilde{t}_{1}$ states in the decoupling regime reads

$$
g_{h \tilde{t}_{1} \tilde{t}_{1}}=\cos 2 \beta M_{Z}^{2}\left[\frac{1}{2} \cos ^{2} \theta_{t}-\frac{2}{3} s_{W}^{2} \cos 2 \theta_{t}\right]+m_{t}^{2}+\frac{1}{2} \sin 2 \theta_{t} m_{t} X_{t}, \sin 2 \theta_{t}=\frac{2 m_{t} X_{t}}{m_{\tilde{t}_{1}}^{2}-m_{\tilde{t}_{2}}^{2}}
$$

In the no-mixing scenario $X_{t} \approx 0$, the coupling above is $\propto m_{t}^{2}$ and the scalar top contribution to the $h g g$ amplitude is small, being damped by a factor $1 / m_{\tilde{t}_{1}}^{2}$ and interferes constructively with the top quark contribution to increase the $g g \rightarrow h$ rate. However, since in the no-mixing scenario $M_{S}=\sqrt{m_{\tilde{t}_{1}} m_{\tilde{t}_{2}}}$ has to be very large for the $h$ boson mass to reach a value $M_{h} \approx 126 \mathrm{GeV}$, the stop contribution to the $h g g$ vertex, $\propto m_{t}^{2} / M_{S}^{2}$, is very small. In the maximal mixing scenario, $X_{t} \approx \sqrt{6} M_{S}$, it is the last component of $g_{h \tilde{t}_{1} \tilde{t}_{1}}$ which dominates and becomes very large, $\propto-\left(m_{t} X_{t} / m_{\tilde{t}_{2}}\right)^{2}$. However, in this case, the large contribution of a light stop to the $h g g$ amplitude interferes destructively with the top quark contribution and the $g g \rightarrow h$ cross section is suppressed. For $m_{\tilde{t}_{1}} \approx 200 \mathrm{GeV}$ and $X_{t} \approx 1 \mathrm{TeV}$, we obtain a factor of two smaller $g g \rightarrow h$ rate. In the case of sbottom squarks, the same situation may occur for large sbottom mixing $X_{b}=A_{b}-\mu \tan \beta$. However, for large value of $M_{S}$, it is more difficult to obtain a small enough $m_{\tilde{b}_{1}}$ state to significantly affect the $g g \rightarrow h$ cross section.

In the case of the $h \gamma \gamma$ decay amplitude, there is the additional SM contribution of the $W$ boson, which is in fact the dominant. Also, it has the opposite sign to that from the top quark and, hence, when stops are light and have a strong mixing, they will tend to increase the $h \gamma \gamma$ amplitude. However, because the $W$ contribution is by far the largest, the stop impact will be much more limited compared to the $g g h$ case and we can expect to have only a $\approx 10 \%$ increase of the $h \rightarrow \gamma \gamma$ decay rate for $m_{\tilde{t}_{1}} \approx 200 \mathrm{GeV}$ and $X_{t} \approx 1 \mathrm{TeV}[51-$ 53]. Therefore, for light and strongly mixed stops, the cross section times branching ratio $\mu_{\gamma \gamma}$ is always smaller unity and relatively light stops do not entail an enhancement of the $\gamma \gamma$ yield. The sbottom contribution to the $h \gamma \gamma$ vertex is also very small, for the same reasons discussed above in the case of the $h g g$ amplitude, and also because of its

\footnotetext{
${ }^{5}$ On should assume $X_{t}$ values such that $A_{t} \lesssim 3 M_{S}$ to avoid dangerous charge and colour breaking minima. In addition, if $X_{t} \gtrsim \sqrt{6} M_{S}$, the radiative corrections to the $h$ boson mass become small again and it would be difficult to attain the value $M_{h} \approx 126 \mathrm{GeV}$.
} 

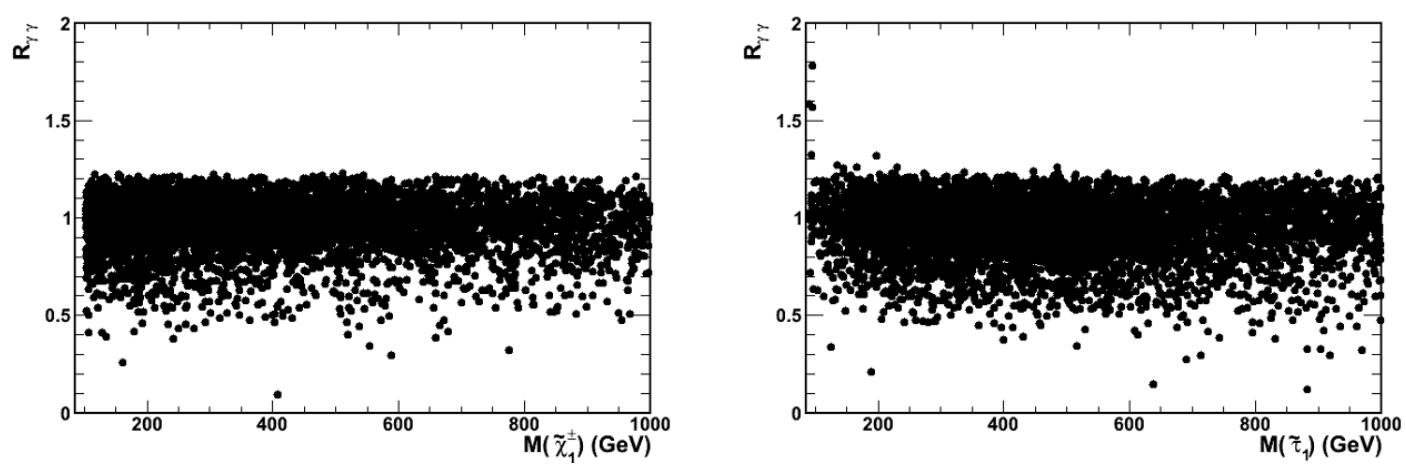

Figure 11. $R_{\gamma \gamma}$ values for a sample of pMSSM points as a function of $m_{\chi_{1}^{ \pm}}$(left) and $\left(m_{\tau_{1}^{ \pm}}\right.$) (right). We impose $R_{b b}>0.9$, to remove the effects due to the changes of the total width through the $b b$ channel.

electric charge, $-\frac{1}{3}$ compared to $+\frac{2}{3}$ for stops. Other charged particles can also contribute to the $h \rightarrow \gamma \gamma$ rate [52]. The charged Higgs bosons have negligible contributions for $m_{H^{ \pm}} \gtrsim 200 \mathrm{GeV}$. Charginos contribute to the $h \gamma \gamma$ vertex and, because of their spin $\frac{1}{2}$ nature, they contribution is only damped by powers of $M_{h} / m_{\chi^{ \pm}}$. However, the $h \chi_{1,2}^{ \pm} \chi_{1,2}^{\mp}$ couplings are similar in nature to those of the LSP given in eq. (3.6) and cannot be strongly enhanced. As a result we expect contributions at most of the order of $10 \%$ even for mass values $m_{\chi_{1}^{ \pm}} \approx 100 \mathrm{GeV}$ (see figure 11). Charged sleptons have in general also little effect on the $h \gamma \gamma$ vertex, with the exception of staus [8]. These behave like the bottom squarks. At very large $\mu \tan \beta$ values, the splitting between the two $\tilde{\tau}$ states becomes significant and their couplings to the $h$ boson large. Since $\tilde{\tau}_{1}$ can have a mass of the order of a few $100 \mathrm{GeV}$, without affecting the value of $M_{h}$, its contribution to the $h \gamma \gamma$ amplitude may be significant for large values of $X_{\tau}$ (see figure 11).

\subsubsection{Impact of the LHC data}

Now, it is interesting to perform a first assessment of the compatibility of the LHC and Tevatron data with the MSSM and analyse the region of parameter favoured by the observed boson mass and rate pattern (see also [7, 104]). Despite the preliminary character of the results reported by the LHC collaborations and the limited statistical accuracy of these first results, the study is a template for future analyses. In this analysis, we compute the $\chi^{2}$ probability on the observable of table 1 for each accepted pMSSM points. For the $b \bar{b}$ and $\tau^{+} \tau^{-}$channels, in which no evidence has been obtained at the LHC, we add the channel contribution to the total $\chi^{2}$ only when their respective $\mu$ value exceeded 1.5 and the pMSSM point becomes increasingly less consistent to the limits reported by CMS. In order to investigate the sensitivity to the inputs, we also compare the results by including or not the $b \bar{b}$, for which a tension exists between the CMS limit and Tevatron results, and the $\tau^{+} \tau^{-}$rate. Figure 12 shows the region of the $\left[X_{t}, m_{\tilde{t}_{1}}\right],\left[X_{b}, m_{\tilde{b}_{1}}\right]$ and $\left[M_{A}, \tan \beta\right]$ parameter space where pMSSM points are compatible with the input $h$ boson mass and observed yields. In particular, we observe an almost complete suppression for low values of the sbottom mixing parameter $X_{b}$. 

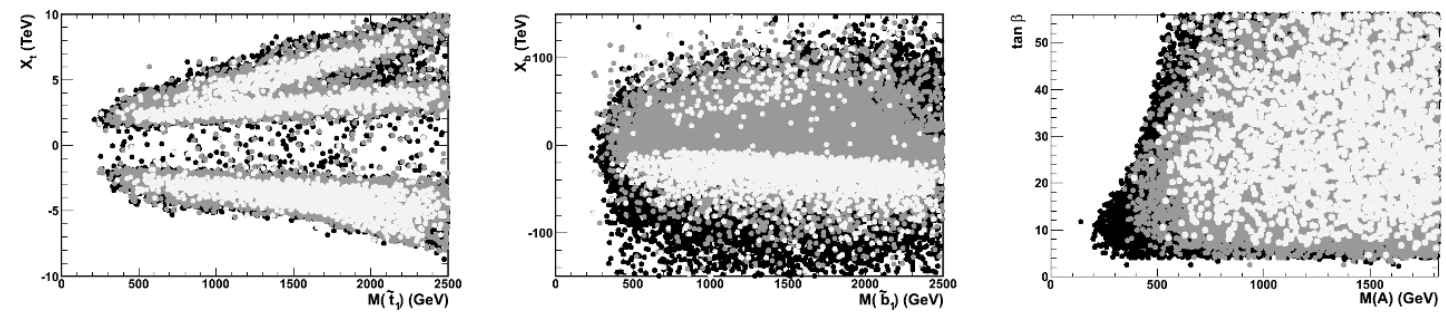

Figure 12. Distributions of the pMSSM points in the $\left[X_{t}, m_{\tilde{t}_{1}}\right]$ (left), $\left[X_{b}, m_{\tilde{b}_{1}}\right]$ (centre) and $\left[M_{A}\right.$, $\tan \beta]$ (right) parameter space. The black dots show the selected pMSSM points, those in light (dark) grey the same points compatible at $68 \%$ (90\%) C.L. with the mass and rate constraints of table 1.

The distributions for some individual parameters which may manifest a sensitivity are presented in figure 13, where each pMSSM point enters with a weight equal to its $\chi^{2}$ probability. Points having a probability below 0.15 are not included. The normalised probability-weighted distributions obtained from this analysis are compared to the normalised frequency distribution for the same observables obtained for the accepted points within the allowed mass region $123<M_{H}<129 \mathrm{GeV}$. We observe that some variables are significantly affected by the constraints applied. Not surprisingly, the observable which exhibits the largest effect is the product $\mu \tan \beta$, for which the data favours large positive values, where the $\gamma \gamma$ branching fraction increases and the $b \bar{b}$ decreases as discussed above. On the contrary, it appears difficult to reconcile an enhancement of both $\mu_{\gamma \gamma}$ and $\mu_{b \bar{b}}$, as would be suggested by the central large value of $\mu_{b \bar{b}}=1.97 \pm 0.72$, recently reported by the Tevatron experiments [5]. Such an enhancement is not observed by the CMS collaboration and the issue is awaiting the first significant evidence of the boson signal in the $b \bar{b}$ final state at the LHC and the subsequent rate determination. The $\tan \beta$ distribution is also shifted towards larger value as an effect of the Higgs mass and rate values. We also observe a significant suppression of pMSSM points with the pseudo-scalar $A$ boson mass below $\sim 450 \mathrm{GeV}$. This is due to the combined effect of the $A \rightarrow \tau^{+} \tau^{-}$direct searches and $B_{s} \rightarrow \mu^{+} \mu^{-}$rate, which constrain the $\left[M_{A}-\tan \beta\right]$ plane to low $\tan \beta$ value for light $A$ masses, by the shift to $\mu \tan \beta$ from the Higgs rates disfavouring the low $\tan \beta$ region and by the suppression of the non-decoupling regime.

In quantitative terms, we observe that $0.06(0.50)$ of the selected pMSSM points are compatible with the constraints given in table 1 at the $68 \%(90 \%)$ confidence level. If we remove the constraint on the upper limit constraint on the $b \bar{b}$ and $\tau^{+} \tau^{-}$rates, the fraction of points accepted at the $90 \%$ C.L. does not change significantly, at 0.56 , but that at the $68 \%$ C.L. doubles to 0.12 . On the contrary, if we replace the CMS upper limit for $\mu_{b b}$ with the $\mu_{b b}$ result of the Tevatron experiments for $M_{H}=125 \mathrm{GeV}$ [5], the fraction of accepted points at $68 \%$ C.L. drops below 0.005 . This highlights the tension which will be created in the pMSSM by a simultaneous excess in the $\gamma \gamma$ and $b \bar{b}$ channels, excess which cannot be adequately described in the pMSSM, as discussed above (see figure 10). 

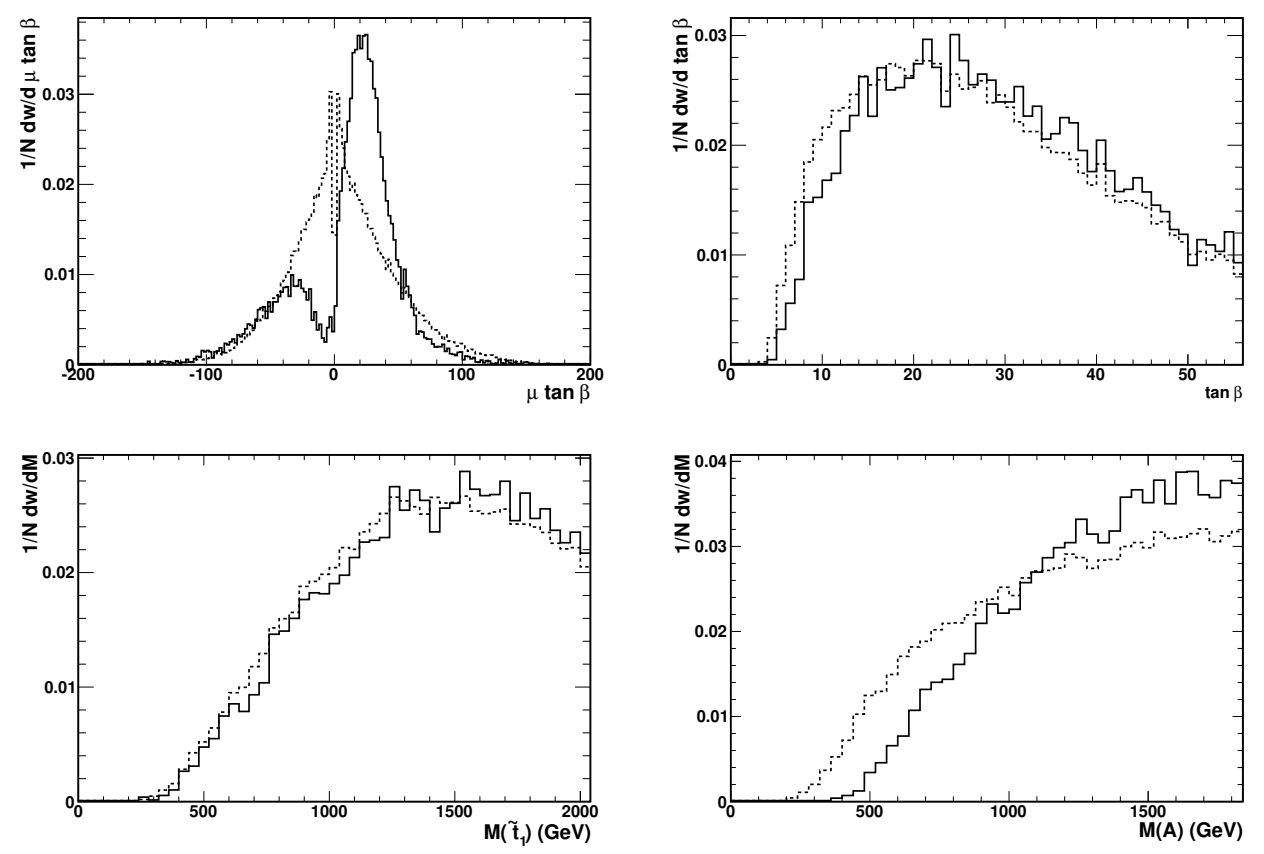

Figure 13. The normalised distribution of the values of the $\mu \tan \beta$ (upper left), $\tan \beta$ (upper right), $M_{\tilde{t}_{1}}$ (lower left) and $M_{A}$ (lower right) variables for the selected pMSSM points (dashed line) compared to the probability density function for the same variables obtained from the $\chi^{2}$ probability corresponding to the mass and rate constraints of table 1 (continuous line). The normalised distributions reflect the biases induced by these constraints.

\section{Conclusions}

The implications of the new boson observation by the ATLAS and CMS collaborations for the phenomenological MSSM have been outlined. The study has been based on broad scans over the pMSSM parameter space where points have been preselected based on constraints from electro-weak and flavour physics, dark matter and searches at LEP2 and the LHC. Various scenarios for the stop mixing parameter $X_{t}$ (maximal, typical and zeromixing) and representative values of the soft SUSY-breaking scale $M_{S}(1,2$ and $3 \mathrm{TeV})$ have been confronted with the Higgs mass range compatible with LHC results, accounting for systematic uncertainties. In order to obtain $M_{h}$ in the mass range $123 \mathrm{GeV} \leq M_{h} \leq$ $129 \mathrm{GeV}$, large values of $M_{S}$ and/or $X_{t}$ are required. In particular, the $M_{h}$ constraints are sensitive to the value of the top quark mass for which the value extracted from the top quark pair production cross section has a more unambiguous definition but larger uncertainties.

The various regimes of the pMSSM Higgs sector have been examined in the $\left[M_{A}, \tan \beta\right]$ parameter. Of these regimes, only the decoupling regime, where the lighter $h$ boson has almost SM-like properties and the heavier Higgs particles decouple from gauge bosons, and the SUSY regime survives all constraints. The anti-decoupling regime where the $H$ state plays the role of the SM Higgs boson, the intense coupling regime in which there are three light states $h, H$ and $A$, the vanishing coupling regime in which the $h$ coupling to bottom quarks or gauge bosons are very strongly suppressed, and most of the intermediate 
coupling regime with relatively low $M_{A}$ and $\tan \beta$ values, are excluded by the present data. In the SUSY regime light superparticles may affect the production and decay rates of the $h$ boson. Light neutralinos may lead to invisible $h$ boson decays, light stop and sbottom quarks affect the $h b \bar{b}$ couplings and the production cross section in the dominant gluongluon fusion mechanism, and light squarks, $\tau$-sleptons and charginos may affect the $h \rightarrow \gamma \gamma$ decay mode.

We have confronted these possibilities with the recent LHC results and find that a significant fraction of pMSSM points in our scan compatible with them, including a possible enhancement of the $\gamma \gamma$ rate. Improved precision in the experimental measurements and sensitivity to the direct searches for the heavier Higgs bosons and supersymmetric particle partners at the LHC will provide the basis for clarifying the relation between the newly discovered scalar sector and physics beyond the Standard Model.

\section{Acknowledgments}

A.A. and F.M. acknowledge partial support from the European Union FP7 ITN INVISIBLES (Marie Curie Actions, PITN-GA-2011-289442). A.D. thanks the CERN TH unit for hospitality.

Open Access. This article is distributed under the terms of the Creative Commons Attribution License which permits any use, distribution and reproduction in any medium, provided the original author(s) and source are credited.

\section{References}

[1] ATLAS collaboration, Combined search for the Standard Model Higgs boson in pp collisions at $\sqrt{s}=7$ TeV with the ATLAS detector, PH-EP-2012-167 (2012).

[2] ATLAS collaboration, F. Gianotti, Status of standard model Higgs searches at ATLAS, talk given at CERN Seminar, July 4, CERN, Switzerland (2012).

[3] CMS collaboration, Observation of a new boson with a mass near $125 \mathrm{GeV}$, CMS-PAS-HIG-12-020 (2012).

[4] CMS collaboration, J. Incandela, Stauts of the CMS SM Higgs search, talk given at CERN Seminar, July 4, CERN, Switzerland (2012).

[5] CDF and D0 collaboration, Updated combination of CDF and D0 searches for standard model Higgs boson production with up to $10.0 \mathrm{fb}^{-1}$ of data, FERMILAB-CONF-12-318-E (2012).

[6] A. Arbey, M. Battaglia, A. Djouadi, F. Mahmoudi and J. Quevillon, Implications of a 125 GeV Higgs for supersymmetric models, Phys. Lett. B 708 (2012) 162 [arXiv:1112.3028] [INSPIRE].

[7] A. Arbey, M. Battaglia and F. Mahmoudi, Constraints on the MSSM from the Higgs sector: a pMSSM study of Higgs searches, $B_{s}^{0} \rightarrow \mu^{+} \mu^{-}$and dark matter direct detection, Eur. Phys. J. C 72 (2012) 1906 [arXiv:1112.3032] [InSPIRE].

[8] M. Carena, S. Gori, N.R. Shah and C.E. Wagner, A $125 \mathrm{GeV}$ SM-like Higgs in the MSSM and the $\gamma \gamma$ rate, JHEP 03 (2012) 014 [arXiv:1112.3336] [INSPIRE]. 
[9] H. Baer, V. Barger and A. Mustafayev, Implications of a 125 GeV Higgs scalar for LHC SUSY and neutralino dark matter searches, Phys. Rev. D 85 (2012) 075010 [arXiv:1112.3017] [INSPIRE].

[10] S. Heinemeyer, O. Stal and G. Weiglein, Interpreting the LHC Higgs search results in the MSSM, Phys. Lett. B 710 (2012) 201 [arXiv:1112.3026] [InSPIRE].

[11] P. Draper, P. Meade, M. Reece and D. Shih, Implications of a $125 \mathrm{GeV}$ Higgs for the MSSM and low-scale SUSY breaking, Phys. Rev. D 85 (2012) 095007 [arXiv:1112.3068] [INSPIRE].

[12] O. Buchmueller et al., Higgs and supersymmetry, Eur. Phys. J. C 72 (2012) 2020 [arXiv:1112.3564] [INSPIRE].

[13] S. Akula, B. Altunkaynak, D. Feldman, P. Nath and G. Peim, Higgs boson mass predictions in SUGRA unification, recent LHC-7 results and dark matter, Phys. Rev. D 85 (2012) 075001 [arXiv: 1112.3645] [INSPIRE].

[14] C. Strege et al., Updated global fits of the CMSSM including the latest LHC SUSY and Higgs searches and XENON100 data, JCAP 03 (2012) 030 [arXiv:1112.4192] [INSPIRE].

[15] C. Beskidt, W. de Boer, D. Kazakov and F. Ratnikov, Where is SUSY?, JHEP 05 (2012) 094 [arXiv: 1202.3366] [INSPIRE].

[16] M. Carena, S. Gori, N.R. Shah, C.E. Wagner and L.-T. Wang, Light stau phenomenology and the Higgs $\gamma \gamma$ rate, JHEP 07 (2012) 175 [arXiv:1205.5842] [INSPIRE].

[17] M.W. Cahill-Rowley, J.L. Hewett, A. Ismail and T.G. Rizzo, The Higgs sector and fine-tuning in the pMSSM, arXiv:1206.5800 [INSPIRE].

[18] MSSM Working Group collaboration, A. Djouadi et al., The minimal supersymmetric standard model: Group summary report, hep-ph/9901246 [INSPIRE].

[19] A. Djouadi, The anatomy of electro-weak symmetry breaking. II. The Higgs bosons in the minimal supersymmetric model, Phys. Rept. 459 (2008) 1 [hep-ph/0503173] [INSPIRE].

[20] M.S. Carena and H.E. Haber, Higgs boson theory and phenomenology, Prog. Part. Nucl. Phys. 50 (2003) 63 [hep-ph/0208209] [INSPIRE].

[21] S. Heinemeyer, W. Hollik and G. Weiglein, Electroweak precision observables in the minimal supersymmetric standard model, Phys. Rept. 425 (2006) 265 [hep-ph/0412214] [INSPIRE].

[22] S. Heinemeyer, MSSM Higgs physics at higher orders, Int. J. Mod. Phys. A 21 (2006) 2659 [hep-ph/0407244] [INSPIRE].

[23] A. Djouadi, The anatomy of electro-weak symmetry breaking. I: The Higgs boson in the standard model, Phys. Rept. 457 (2008) 1 [hep-ph/0503172] [INSPIRE].

[24] Y. Okada, M. Yamaguchi and T. Yanagida, Upper bound of the lightest Higgs boson mass in the minimal supersymmetric standard model, Prog. Theor. Phys. 85 (1991) 1 [INSPIRE].

[25] J.R. Ellis, G. Ridolfi and F. Zwirner, Radiative corrections to the masses of supersymmetric Higgs bosons, Phys. Lett. B 257 (1991) 83 [INSPIRE].

[26] H.E. Haber and R. Hempfling, Can the mass of the lightest Higgs boson of the minimal supersymmetric model be larger than $m(Z)$ ?, Phys. Rev. Lett. 66 (1991) 1815 [INSPIRE].

[27] M.S. Carena, S. Heinemeyer, C. Wagner and G. Weiglein, Suggestions for benchmark scenarios for MSSM Higgs boson searches at hadron colliders, Eur. Phys. J. C 26 (2003) 601 [hep-ph/0202167] [INSPIRE]. 
[28] B. Allanach, A. Djouadi, J. Kneur, W. Porod and P. Slavich, Precise determination of the neutral Higgs boson masses in the MSSM, JHEP 09 (2004) 044 [hep-ph/0406166] [INSPIRE].

[29] G. Degrassi, P. Slavich and F. Zwirner, On the neutral Higgs boson masses in the MSSM for arbitrary stop mixing, Nucl. Phys. B 611 (2001) 403 [hep-ph/0105096] [INSPIRE].

[30] A. Brignole, G. Degrassi, P. Slavich and F. Zwirner, On the two loop sbottom corrections to the neutral Higgs boson masses in the MSSM, Nucl. Phys. B 643 (2002) 79 [hep-ph/0206101] [INSPIRE].

[31] A. Brignole, G. Degrassi, P. Slavich and F. Zwirner, On the $O\left(\alpha_{t}^{2}\right)$ two loop corrections to the neutral Higgs boson masses in the MSSM, Nucl. Phys. B 631 (2002) 195 [hep-ph/0112177] [INSPIRE].

[32] A. Djouadi, J.-L. Kneur and G. Moultaka, SuSpect: a Fortran code for the supersymmetric and Higgs particle spectrum in the MSSM, Comput. Phys. Commun. 176 (2007) 426 [hep-ph/0211331] [INSPIRE].

[33] B. Allanach, SOFTSUSY: a program for calculating supersymmetric spectra, Comput. Phys. Commun. 143 (2002) 305 [hep-ph/0104145] [INSPIRE].

[34] S. Heinemeyer, W. Hollik and G. Weiglein, QCD corrections to the masses of the neutral CP-even Higgs bosons in the MSSM, Phys. Rev. D 58 (1998) 091701 [hep-ph/9803277] [INSPIRE].

[35] S. Heinemeyer, W. Hollik and G. Weiglein, The masses of the neutral CP-even Higgs bosons in the MSSM: accurate analysis at the two loop level, Eur. Phys. J. C 9 (1999) 343 [hep-ph/9812472] [INSPIRE].

[36] S. Heinemeyer, W. Hollik and G. Weiglein, FeynHiggs: a program for the calculation of the masses of the neutral CP even Higgs bosons in the MSSM, Comput. Phys. Commun. 124 (2000) 76 [hep-ph/9812320] [INSPIRE].

[37] P. Kant, R. Harlander, L. Mihaila and M. Steinhauser, Light MSSM Higgs boson mass to three-loop accuracy, JHEP 08 (2010) 104 [arXiv: 1005.5709] [INSPIRE].

[38] H.E. Haber and Y. Nir, The decay $Z \rightarrow A^{0} A^{0}$ neutrino anti-neutrino and $e^{+} e^{-} \rightarrow A^{0} A^{0} z$ in two Higgs doublet models, Phys. Lett. B 306 (1993) 327 [hep-ph/9302228] [INSPIRE].

[39] H.E. Haber, Challenges for nonminimal Higgs searches at future colliders, hep-ph/9505240 [INSPIRE].

[40] J. Gunion, A. Stange and S. Willenbrock, Weakly coupled Higgs bosons, hep-ph/9602238 [INSPIRE].

[41] E. Boos, A. Djouadi, M. Muhlleitner and A. Vologdin, The MSSM Higgs bosons in the intense coupling regime, Phys. Rev. D 66 (2002) 055004 [hep-ph/0205160] [INSPIRE].

[42] E. Boos, V. Bunichev, A. Djouadi and H. Schreiber, Prospects of mass measurements for neutral MSSM Higgs bosons in the intense-coupling regime at a linear collider, Phys. Lett. B 622 (2005) 311 [hep-ph/0412194] [INSPIRE].

[43] A. Djouadi and Y. Mambrini, The Higgs intense-coupling regime in constrained SUSY models and its astrophysical implications, JHEP 12 (2006) 001 [hep-ph/0609234] [INSPIRE].

[44] E. Boos, A. Djouadi and A. Nikitenko, Detection of the neutral MSSM Higgs bosons in the 
intense coupling regime at the LHC, Phys. Lett. B 578 (2004) 384 [hep-ph/0307079] [INSPIRE].

[45] H. Baer and J.D. Wells, Trilepton Higgs signal at hadron colliders, Phys. Rev. D 57 (1998) 4446 [hep-ph/9710368] [INSPIRE].

[46] W. Loinaz and J.D. Wells, Higgs boson interactions in supersymmetric theories with large $\tan \beta$, Phys. Lett. B 445 (1998) 178 [hep-ph/9808287] [INSPIRE].

[47] K. Babu and C.F. Kolda, Signatures of supersymmetry and Yukawa unification in Higgs decays, Phys. Lett. B 451 (1999) 77 [hep-ph/9811308] [INSPIRE].

[48] M.S. Carena, S. Mrenna and C. Wagner, The complementarity of LEP, the Tevatron and the CERN LHC in the search for a light MSSM Higgs boson, Phys. Rev. D 62 (2000) 055008 [hep-ph/9907422] [INSPIRE].

[49] S. Heinemeyer, W. Hollik and G. Weiglein, Decay widths of the neutral CP even MSSM Higgs bosons in the Feynman diagrammatic approach, Eur. Phys. J. C 16 (2000) 139 [hep-ph/0003022] [INSPIRE].

[50] D. Noth and M. Spira, Supersymmetric Higgs Yukawa couplings to bottom quarks at next-to-next-to-leading order, JHEP 06 (2011) 084 [arXiv:1001.1935] [INSPIRE].

[51] A. Djouadi, Squark effects on Higgs boson production and decay at the LHC, Phys. Lett. B 435 (1998) 101 [hep-ph/9806315] [INSPIRE].

[52] A. Djouadi, V. Driesen, W. Hollik and J.I. Illana, The coupling of the lightest SUSY Higgs boson to two photons in the decoupling regime, Eur. Phys. J. C 1 (1998) 149 [hep-ph/9612362] [INSPIRE].

[53] A. Arvanitaki and G. Villadoro, A non standard model Higgs at the LHC as a sign of naturalness, JHEP 02 (2012) 144 [arXiv:1112.4835] [INSPIRE].

[54] H.K. Dreiner, J.S. Kim and O. Lebedev, First LHC constraints on neutralinos, Phys. Lett. B 715 (2012) 199 [arXiv:1206.3096] [InSPIRE].

[55] A. Djouadi, P. Janot, J. Kalinowski and P. Zerwas, SUSY decays of Higgs particles, Phys. Lett. B 376 (1996) 220 [hep-ph/9603368] [INSPIRE].

[56] A. Djouadi, J. Kalinowski and P. Zerwas, Two and three-body decay modes of SUSY Higgs particles, Z. Phys. C 70 (1996) 435 [hep-ph/9511342] [INSPIRE].

[57] A. Djouadi, J. Kalinowski, P. Ohmann and P. Zerwas, Heavy SUSY Higgs bosons at $e^{+} e^{-}$ linear colliders, Z. Phys. C 74 (1997) 93 [hep-ph/9605339] [INSPIRE].

[58] A. Djouadi, Impact of the SUSY decays on the search for the MSSM Higgs bosons at the LHC, Mod. Phys. Lett. A 14 (1999) 359 [hep-ph/9903382] [INSPIRE].

[59] A. Djouadi, J. Kalinowski and M. Spira, HDECAY: a program for Higgs boson decays in the standard model and its supersymmetric extension, Comput. Phys. Commun. 108 (1998) 56 [hep-ph/9704448] [INSPIRE].

[60] D. Albornoz Vasquez, G. Bélanger, R. Godbole and A. Pukhov, The Higgs boson in the MSSM in light of the LHC, Phys. Rev. D 85 (2012) 115013 [arXiv:1112.2200] [InSPIRE].

[61] R.V. Harlander and W.B. Kilgore, Higgs boson production in bottom quark fusion at next-to-next-to leading order, Phys. Rev. D 68 (2003) 013001 [hep-ph/0304035] [INSPIRE].

[62] Particle Data Group collaboration, K. Nakamura et al., Review of particle physics, J. Phys. G 37 (2010) 075021 [INSPIRE]. 
[63] A. Arbey, M. Battaglia and F. Mahmoudi, Implications of LHC searches on SUSY particle spectra: the pMSSM parameter space with neutralino dark matter, Eur. Phys. J. C 72 (2012) 1847 [arXiv: 1110.3726] [INSPIRE].

[64] M. Muhlleitner, A. Djouadi and Y. Mambrini, SDECAY: a Fortran code for the decays of the supersymmetric particles in the MSSM, Comput. Phys. Commun. 168 (2005) 46 [hep-ph/0311167] [INSPIRE].

[65] C. Boehm, A. Djouadi and Y. Mambrini, Decays of the lightest top squark, Phys. Rev. D 61 (2000) 095006 [hep-ph/9907428] [INSPIRE].

[66] A. Djouadi and Y. Mambrini, Three body decays of top and bottom squarks, Phys. Rev. D 63 (2001) 115005 [hep-ph/0011364] [INSPIRE].

[67] A. Djouadi, Y. Mambrini and M. Muhlleitner, Chargino and neutralino decays revisited, Eur. Phys. J. C 20 (2001) 563 [hep-ph/0104115] [INSPIRE].

[68] F. Mahmoudi, SuperIso: a program for calculating the isospin asymmetry of $B \rightarrow K^{*} \gamma$ in the MSSM, Comput. Phys. Commun. 178 (2008) 745 [arXiv:0710.2067] [InSPIRE].

[69] F. Mahmoudi, SuperIso v2.3: a program for calculating flavor physics observables in supersymmetry, Comput. Phys. Commun. 180 (2009) 1579 [arXiv:0808.3144] [INSPIRE].

[70] A. Arbey and F. Mahmoudi, SuperIso relic: a program for calculating relic density and flavor physics observables in supersymmetry, Comput. Phys. Commun. 181 (2010) 1277 [arXiv: 0906.0369] [INSPIRE].

[71] M. Spira, HIGLU and HDECAY: programs for Higgs boson production at the LHC and Higgs boson decay widths, Nucl. Instrum. Meth. A 389 (1997) 357 [hep-ph/9610350] [INSPIRE].

[72] M. Spira, HIGLU: a program for the calculation of the total Higgs production cross-section at hadron colliders via gluon fusion including QCD corrections, hep-ph/9510347 [INSPIRE].

[73] M. Spira, A. Djouadi, D. Graudenz and P. Zerwas, Higgs boson production at the LHC, Nucl. Phys. B 453 (1995) 17 [hep-ph/9504378] [INSPIRE].

[74] CDF collaboration, T. Aaltonen et al., Search for $B_{s} \rightarrow \mu^{+} \mu^{-}$and $B_{d} \rightarrow \mu^{+} \mu^{-}$Decays with CDF II, Phys. Rev. Lett. 107 (2011) 239903 [Phys. Rev. Lett. 107 (2011) 191801] [arXiv:1107.2304] [INSPIRE].

[75] LHCB collaboration, R. Aaij et al., Strong constraints on the rare decays $B_{s} \rightarrow \mu^{+} \mu^{-}$and $B^{0} \rightarrow \mu^{+} \mu^{-}$, Phys. Rev. Lett. 108 (2012) 231801 [arXiv:1203.4493] [INSPIRE].

[76] CMS collaboration, S. Chatrchyan et al., Search for $B_{s}^{0} t o \mu^{+} \mu^{-}$and $B^{0}$ to $\mu^{+} \mu^{-}$decays, JHEP 04 (2012) 033 [arXiv: 1203.3976] [INSPIRE].

[77] A. Akeroyd, F. Mahmoudi and D.M. Santos, The decay $B_{s} \rightarrow \mu^{+} \mu^{-}$: updated SUSY constraints and prospects, JHEP 12 (2011) 088 [arXiv:1108.3018] [INSPIRE].

[78] F. Mahmoudi, S. Neshatpour and J. Orloff, Supersymmetric constraints from $B_{s} \rightarrow \mu^{+} \mu^{-}$ and $B \rightarrow K * \mu^{+} \mu^{-}$observables, JHEP 08 (2012) 092 [arXiv:1205.1845] [INSPIRE].

[79] T. Hurth and F. Mahmoudi, The minimal flavour violation benchmark in view of the latest LHCb data, Nucl. Phys. B 865 (2012) 461 [arXiv:1207.0688] [INSPIRE].

[80] XENON100 collaboration, E. Aprile et al., Dark matter results from 100 live days of XENON100 data, Phys. Rev. Lett. 107 (2011) 131302 [arXiv:1104.2549] [INSPIRE]. 
[81] WMAP collaboration, E. Komatsu et al., Seven-year Wilkinson Microwave Anisotropy Probe (WMAP) observations: cosmological interpretation, Astrophys. J. Suppl. 192 (2011) 18 [arXiv: 1001.4538] [INSPIRE].

[82] A. Arbey and F. Mahmoudi, SUSY constraints from relic density: High sensitivity to pre-BBN expansion rate, Phys. Lett. B 669 (2008) 46 [arXiv:0803.0741] [InSPIRE].

[83] A. Arbey and F. Mahmoudi, SUSY constraints, relic density and very early universe, JHEP 05 (2010) 051 [arXiv:0906.0368] [INSPIRE].

[84] ATLAS collaboration, G. Aad et al., Search for squarks and gluinos using final states with jets and missing transverse momentum with the ATLAS detector in $\sqrt{s}=7$ TeV proton-proton collisions, Phys. Lett. B 710 (2012) 67 [arXiv:1109.6572] [InSPIRE].

[85] CMS collaboration, S. Chatrchyan et al., Search for supersymmetry at the LHC in events with jets and missing transverse energy, Phys. Rev. Lett. 107 (2011) 221804 [arXiv:1109.2352] [INSPIRE].

[86] ATLAS collaboration, G. Aad et al., Search for scalar bottom pair production with the ATLAS detector in pp collisions at $\sqrt{s}=7$ TeV, Phys. Rev. Lett. 108 (2012) 181802 [arXiv:1112.3832] [INSPIRE].

[87] ATLAS collaboration, G. Aad et al., Search for neutral MSSM Higgs bosons decaying to $\tau^{+} \tau^{-}$pairs in proton-proton collisions at $\sqrt{s}=7$ TeV with the ATLAS detector, Phys. Lett. B 705 (2011) 174 [arXiv:1107.5003] [INSPIRE].

[88] CMS collaboration, S. Chatrchyan et al., Search for neutral Higgs bosons decaying to $\tau$ pairs in pp collisions at $\sqrt{s}=7 \mathrm{TeV}$, Phys. Lett. B 713 (2012) 68 [arXiv:1202.4083] [INSPIRE].

[89] ATLAS collaboration, G. Aad et al., Search for charged Higgs bosons decaying via $H^{+} \rightarrow \tau \nu$ in top quark pair events using pp collision data at $\sqrt{s}=7$ TeV with the ATLAS detector, JHEP 06 (2012) 039 [arXiv:1204.2760] [INSPIRE].

[90] ATLAS collaboration, Observation of an excess of events in the search for the standard model Higgs boson in the $\gamma-\gamma$ channel with the ATLAS detector, ATLAS-CONF-2012-091 (2012).

[91] CMS collaboration, Evidence for a new state decaying into two photons in the search for the standard model Higgs boson in pp collisions, CMS-PAS-HIG-2012-015 (2012).

[92] ATLAS collaboration, Observation of an excess of events in the search for the standard model Higgs boson in the $H \rightarrow Z Z^{(*)} \rightarrow 4 \ell$ channel with the ATLAS detector, ATLAS-CONF-2012-092 (2012).

[93] CMS collaboration, Evidence for a new state in the search for the standard model Higgs boson in the $H \rightarrow Z Z \rightarrow 4$ leptons channel in pp collisions at $\sqrt{s}=7$ and 8 TeV, CMS-PAS-HIG-2012-016 (2012).

[94] CMS collaboration, Search for the standard model Higgs boson produced in association with $W$ or $Z$ bosons, and decaying to bottom quarks for ICHEP 2012, CMS-PAS-HIG-2012-019 (2012).

[95] CMS collaboration, Search for a standard model Higgs bosons decaying to $\tau$ pairs in pp collisions, CMS-PAS-HIG-2012-018 (2012).

[96] LHC Higgs Cross Section Working Group collaboration, S. Dittmaier et al., Handbook of LHC Higgs cross sections: 1. Inclusive observables, arXiv:1101.0593 [INSPIRE]. 
[97] J. Baglio and A. Djouadi, Higgs production at the LHC, JHEP 03 (2011) 055 [arXiv: 1012.0530] [INSPIRE].

[98] Tevatron Electroweak Working Group, CDF and D0 collaboration, Combination of CDF and D0 results on the mass of the top quark using up to $5.8 \mathrm{fb}^{-1}$ of data, arXiv: 1107.5255 [INSPIRE].

[99] S. Alekhin, A. Djouadi and S. Moch, The top quark and Higgs boson masses and the stability of the electroweak vacuum, Phys. Lett. B 716 (2012) 214 [arXiv:1207.0980] [INSPIRE].

[100] H. Baer, A. Belyaev, T. Krupovnickas and X. Tata, Linear collider capabilities for supersymmetry in dark matter allowed regions of the mSUGRA model, JHEP 02 (2004) 007 [hep-ph/0311351] [INSPIRE].

[101] H. Baer, T. Krupovnickas and X. Tata, Two photon background and the reach of a linear collider for supersymmetry in WMAP favored coannihilation regions, JHEP 06 (2004) 061 [hep-ph/0405058] [INSPIRE].

[102] A. Djouadi, M. Drees and J.-L. Kneur, Neutralino dark matter in mSUGRA: reopening the light Higgs pole window, Phys. Lett. B 624 (2005) 60 [hep-ph/0504090] [INSPIRE].

[103] A. Arbey, M. Battaglia and F. Mahmoudi, Light neutralino dark matter in the pMSSM: implications of LEP, LHC and dark matter searches on SUSY particle spectra, arXiv: 1205.2557 [INSPIRE].

[104] R. Benbrik et al., Confronting the MSSM and the NMSSM with the discovery of a signal in the two photon channel at the LHC, arXiv:1207.1096 [INSPIRE]. 